\title{
PIV measurements of a plane wall jet in a confined space at transitional slot Reynolds numbers
}

\author{
T. van Hooff - B. Blocken $\cdot$ T. Defraeye $\cdot$ \\ J. Carmeliet • G. J. F. van Heijst
}

Received: 3 November 2011/Revised: 10 March 2012/Accepted: 5 April 2012/Published online: 24 April 2012

(C) The Author(s) 2012. This article is published with open access at Springerlink.com

\begin{abstract}
Wall jets are important for a wide variety of engineering applications, including ventilation of confined spaces and cooling and drying processes. Although a lot of experimental studies have been devoted to wall jets, many of these have focused on laminar or turbulent wall jets. There is a lack of experimental data on transitional wall jets, especially transitional wall jets released into a confined space or enclosure. This paper presents flow visualizations and high-resolution Particle Image Velocimetry measurements of isothermal transitional plane wall jets
\end{abstract}

T. van Hooff $(\bowtie) \cdot$ B. Blocken

Building Physics and Services, Eindhoven University

of Technology, P.O. Box 513, 5600 MB Eindhoven,

The Netherlands

e-mail: t.a.j.v.hooff@tue.nl

T. van Hooff · T. Defraeye

Division of Building Physics, Katholieke Universiteit Leuven,

Kasteelpark Arenberg 40, P.O. Box 2447, 3001 Leuven, Belgium

T. Defraeye

VCBT/MeBioS, Department of Biosystems, Katholieke Universiteit Leuven, Willem de Croylaan 42, P.O. Box 2428, 3001 Leuven, Belgium

J. Carmeliet

Chair of Building Physics, Swiss Federal Institute of Technology

Zürich (ETH), Wolfgang-Pauli-Strasse 15, 8093 Zürich,

Switzerland

J. Carmeliet

Laboratory for Building Science and Technology, Swiss Federal

Laboratories for Materials Testing and Research (Empa),

Überlandstrasse 129, 8600 Dübendorf, Switzerland

G. J. F. van Heijst

Fluid Dynamics Laboratory, Eindhoven University

of Technology, P.O. Box 513, 5600 MB Eindhoven,

The Netherlands injected through a rectangular slot in a confined space. As opposed to many previous studies, not only the wall jet region but also the recirculation region in the remainder of the enclosure is analyzed. The data and analysis in this paper provide new insights into the behavior of transitional plane wall jets in a confined space and will be useful for the validation of numerical simulations of this type of jets.

\section{Introduction}

The dynamics of air jets have been studied extensively in the past decades. Air jets are important for a wide range of engineering applications and are-among others-used for ventilation of buildings, ships and airplanes and for drying and cooling processes. Knowledge of the flow development and mixing characteristics of the jet is, therefore, of primary importance. Previous studies have dealt extensively with the development of turbulent round, plane and wall jets, both experimentally and numerically. However, to the knowledge of the authors, experiments on transitional jets are relatively scarce, especially for transitional plane wall jets issued into a confined space. Providing and analyzing such data are the main objectives of this paper. First, an overview of past studies on wall jets and transitional jets is provided. The findings from these studies will be used to support the analysis of the experiments in this paper.

A wall jet is a jet that is confined on one side by a wall. It can be subdivided in an inner region and an outer region. The inner region, or inner layer, runs from the wall to the point of maximum velocity $U_{M}$ and is similar to a wall boundary layer, whereas the outer region, or outer layer, consists of the remainder of the wall jet and can be seen as a free shear layer. A formal description of the wall jet is given by Launder and Rodi (1981): “A wall jet may be 
defined as a shear flow directed along a wall where, by virtue of the initially supplied momentum, at any station, the streamwise velocity over some region within the shear flow exceeds that in the external stream." Wall jets can be subdivided in two-dimensional (plane) and three-dimensional wall jets. Plane wall jets are bounded by walls on the lateral sides of the jet, which prevent its lateral expansion, whereas three-dimensional wall jets can also grow in the lateral direction and are, therefore, more complex.

The first reported experimental study of a wall jet was conducted by Förthmann (1934). Later, turbulent wall jets were experimentally investigated by Bakke (1957), Sigalla (1958), Bradshaw and Gee (1960) and Schwarz and Cosart (1961). An extensive overview of experimental work until 1980 is given by Launder and Rodi (1981). Wygnanski et al. (1992), Hsiao and Sheu (1994, 1996), Gogineni and Shih (1997), Amitay and Cohen (1997), Eriksson et al. (1998) are just a few examples of studies in which the wall jet was experimentally analyzed during the last decades of the twentieth century.

A non-exhaustive overview of numerical work until the beginning of the 1980s is presented by Launder and Rodi (1983). After 1983, numerical work on wall jets has been performed by, among others, Conlon and Lichter (1995), Gogineni et al. (1999), Davidson et al. (2000), Bhattacharjee and Loth (2004), Wernz and Fasel (2007), Ahlman et al. (2009) and Balabel and El-Askary (2011). Note that unless mentioned otherwise, all studies mentioned above and below were conducted for wall jets in a more or less quiescent flow, that is, a flow domain that is large enough to prevent or minimize the disturbing effects of a secondary flow, which is in contrast to the studied flow pattern presented in this paper (Fig. 1).

Experimental and numerical analyses of turbulent wall jets in a confined space were reported by Moureh and Flick
(2003, 2005). The focus of these studies was on the wall jet characteristics and its decay and detachment from the wall. The authors concluded that the separation of the wall jet from the top surface was caused by an adverse pressure gradient as a result of the confinement of the flow. The location of jet detachment experienced an intermittent behavior, since it oscillated around an average position (Moureh and Flick 2003). In this area, the velocity measurements were characterized by weak fluctuations in the order of $1 \mathrm{~Hz}$. As a result of the intermittent behavior of the location of jet detachment, there was a dynamic interaction between the wall jet flow and the corner recirculation. Consequently, the zones occupied by these two flows expanded and narrowed at the rate of these fluctuations.

Although a lot of experimental studies have been performed for laminar and turbulent wall jets, only a few have focused on transitional wall jets. One of the first experimental studies on transitional plane wall jets was conducted by Bajura and Catalano (1975). They found that the transition from laminar to turbulent flow generally occurred in the following five stages: (1) formation of discrete vortices in outer region, (2) pairing of two or more vortices in the outer region, coupled with the possible pairing of vortex-like motions in the inner region wall boundary layer, (3) lifting-off of the wall jet flow into the ambient fluid, (4) dispersion of the lifted-off flow field by threedimensional turbulent motions and (5) re-laminarization of the upstream flow, until another vortex pairing occurs. Lichter et al. (1992) experimentally analyzed the separation and vortex development of a wall jet in a stratified tank. They observed the organization of an asymmetric dipole after separation from the wall. Hsiao and Sheu (1994) studied the behavior of double row vortical structures in the near-field region of a plane wall jet. Flow visualizations and hot-wire measurements were performed

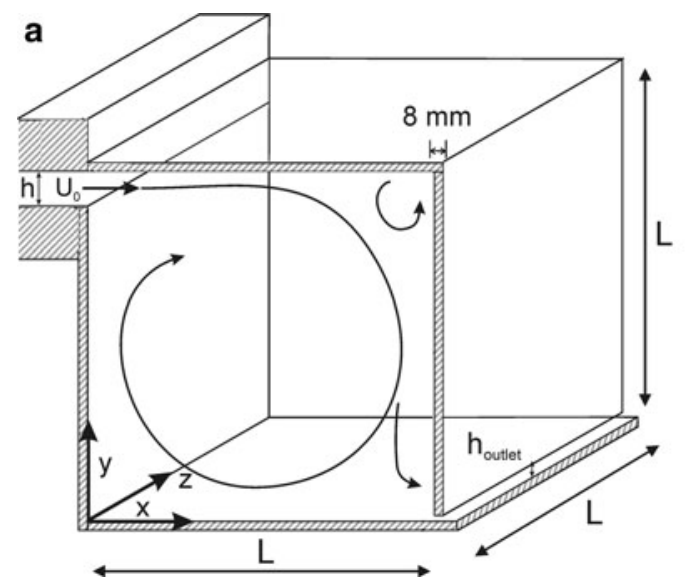

Fig. 1 a Three-dimensional representation of the experimental setup, depicting the coordinate system, the inlet velocity $U_{0}$, the inlet height $h$, the outlet height $h_{\text {outlet }}$ and the dimensions of the test section $L^{3}$. b Two-dimensional schematic representation of the plane wall jet

b

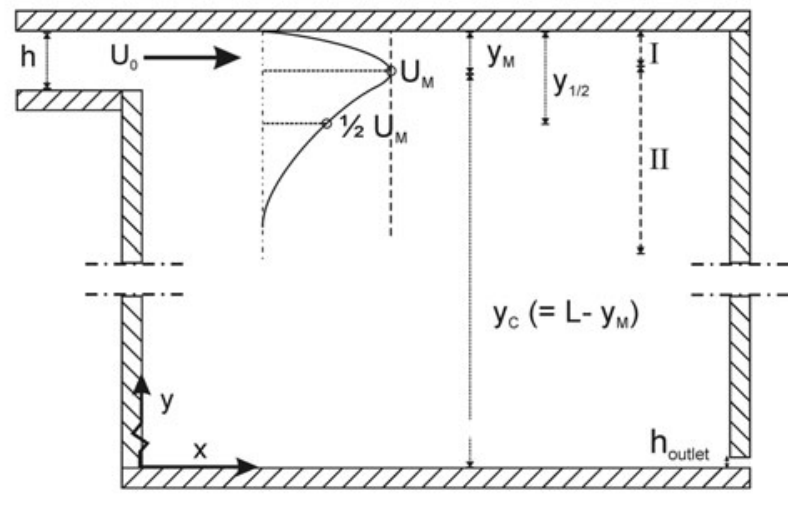

with I the inner region, II the outer region, $U_{M}$ the maximum velocity, $y_{M}$ the distance from the top wall to the location of $U_{M}, y_{C}$ the distance from the bottom wall to the location of $U_{M}$ and $\mathrm{y}_{1 / 2}$ the location of $1 / 2 U_{M}$ in the outer region 
to analyze vortex formation in the inner and outer region and vortex lift-off from the wall. Gogineni and Shih (1997) performed flow visualizations, Particle Image Velocimetry (PIV) and surface pressure measurements to study the vortex pairing and jet detachment associated with the transition of plane wall jets. From their measurements, they concluded that the boundary layer detaches from the wall as a result of a local adverse pressure gradient induced by the passage of a vortex structure in the outer region of the wall jet.

The studies discussed above were all conducted either for turbulent plane wall jets with or without confinement or for transitional plane wall jets without confinement, that is, which are not strongly influenced by a secondary flow. This is in contrast to the study in the present paper, in which a recirculation flow driven by a transitional wall jet dominates the flow in the remainder of the test section. To the knowledge of the authors, only a few studies have been conducted for transitional plane wall jets that are influenced by a secondary flow resulting from a restricted size of the test section (confined flow). Nielsen et al. (2000) and Topp et al. (2000) performed hot-sphere measurements of room air flow resulting from a plane wall jet at low Reynolds numbers. Wang and Chen $(2009,2010)$ performed point measurements of air flow in a simplified model of an airline cabin using hot-sphere and ultrasonic anemometers. The main objective of these measurements was to establish benchmark data to validate numerical flow simulations.

In addition to these wall jet studies, experiments were also performed for transitional flow in free plane jets (Sato 1960; Sato and Sakao 1964; Beavers and Wilson 1970; Mumford 1982; Lemieux and Oosthuizen 1985; Namer and Ötügen 1988). Namer and Ötügen (1988) stated that there is strong evidence that large vortical structures control the initial jet growth. Immediately downstream of the inlet, unstable laminar shear layers break down and form vortices that carry irrotational ambient fluid into the jet and thereby induce mixing by wrapping the ambient fluid about themselves. They also indicated the dependence of jet mixing, spread rate and centerline decay on the jet Reynolds number, but found that the Strouhal number, based on the vortex formation frequency in the outer region, was independent of $R e$ for $R e$-values ranging from 1,000 to 7,000. Suresh et al. (2008) studied the transitional characteristics of plane jets for $R e$ in the range from 250 to 6,250 . They showed that jet spread decreases with $R e$ due to the dominance of finer scales in higher $R e$ jets and large-scale structures in the outer shear layer region for low $R e$ jets $(R e<2,000)$. Furthermore, they concluded that the Strouhal number is $R e$-dependent for $R e<2,000$. Additionally, transitional round jets were studied by Angioletti et al. (2003), O’Neill et al. (2004), Kwon and Seo (2005) and Todde et al. (2009).
Not only studies on transitional jets are relevant sources of information. Also studies of vortex dynamics in confined spaces can contribute to the understanding of vortex formation, advection and decay in an enclosure. For example, van Heijst et al. (1990) experimentally studied the spin-up process in a rectangular container. Konijnenberg et al. (1994) also studied the spin-up process in a rectangular tank, both experimentally and numerically. Wells et al. (2007) performed experiments and numerical simulations to investigate the production of small-scale vorticity near no-slip sidewalls of a container and to study the formation and decay of wall-generated quasi-two-dimensional vortical structures.

The present paper reports flow visualizations and PIV measurements of a transitional plane wall jet issued into a confined space. The study is motivated by the fact that most plane wall jet studies in the past have been conducted for plane wall jets in relatively large enclosures and/or with slot Reynolds numbers (i.e., $R e$ based on inlet height) corresponding with a turbulent regime. As opposed to many previous studies, this study will not only focus on the wall jet region but also on the recirculation region in the remainder of the enclosure. One of the practical situations in which this type of flow is important is the ventilation of enclosures. The use of a wall jet to create a recirculation region that dilutes the air in a confined space is one of the two major ventilation principles that are used to maintain a healthy, energy-efficient and comfortable indoor climate in buildings, ships, planes, etc. (e.g., Nielsen 1974; Etheridge and Sandberg 1996; Awbi 2003; Chen 2009). In order to minimize the risk of discomfort of the occupants, velocities inside an enclosure should be kept relatively low. As a result, the jet flow might become transitional at these low velocities (low $R e$-values). First, the experimental setup is described in Sect. 2. The flow visualizations are addressed in Sect. 3. A description of the PIV measurement setup is given in Sect. 4, after which the results of the PIV measurements are analyzed in Sect. 5. Discussion (Sect. 6) and conclusions (Sect. 7) conclude this paper.

\section{Experimental setup}

A water-filled experimental model has been built to perform flow visualizations and PIV measurements (Fig. 1a). It consists of (1) a water column to drive the flow by hydrostatic pressure; (2) a flow conditioning section; (3) a cubic test section having edges of $L=0.3 \mathrm{~m}$ constructed from glass plates with a thickness of $8 \mathrm{~mm}$; and (4) an overflow. The conditioning section in front of the inlet consists of one honeycomb, three screens and a contraction to obtain a uniform water flow at the inlet and to minimize the turbulence level. More information on the experimental setup can be found in van Hooff et al. (2012). 
The coordinate system is made dimensionless: $x^{\prime}=x / L$, $y^{\prime}=y / L, z^{\prime}=z / L$ (Fig. 1a). The inlet width $(w)$ is $0.3 \mathrm{~m}$ $\left(w^{\prime}=w / L=1\right)$, and the dimensionless inlet height $\left(h^{\prime}=h / L\right)$ can be varied from 0 to 0.1 . For this study, $h^{\prime}$ is fixed at 0.1 . The height of the outlet is fixed at $h_{\text {outlet }}^{\prime}=0.0167$. The slot Reynolds number is defined based on the inlet height as $R e=R e_{\text {slot }}=U_{0} h / v$, with $U_{0}$ the area-averaged inlet velocity based on the volume flow rate through the inlet and $v$ the kinematic viscosity at room temperature $\left(\approx 20^{\circ} \mathrm{C}\right)$. The maximum local velocity $U_{M}$ (Fig. 1b) is used to make the velocities non-dimensional $\left(U / U_{M}\right)$. Note that $U_{M}$ is defined as the local maximum time-averaged $x$-velocity and thus varies with $x$. Furthermore, $U_{M}$ varies with $R e$; since $R e$ is based on the inlet velocity $U_{0}$, the maximum velocities $\left(U_{M}\right)$ are higher for higher values of $R e$. The distance from the top wall to the location of $U_{M}$ is $y_{M}^{\prime}\left(=y_{M} / L\right)$, whereas $y_{C}^{\prime}=y_{C} / L$, with $y_{C}$ the distance from the bottom wall to the location of $U_{M}$. The distance to the location of $1 / 2 U_{M}$ in the outer region is denoted as $y_{1 / 2}$. A useful quantity to characterize the flow in the midplane $z^{\prime}=0.5$ is the $z$-component of the vorticity, defined as

$\omega_{z} \equiv \frac{\partial v}{\partial x}-\frac{\partial u}{\partial y}$

which is non-dimensionalized as $\omega^{\prime}{ }_{z}=\omega_{z} h / U_{M}$. Finally, the Strouhal number is defined as $S t=(f h) / U_{M}$, with $f$ the vortex formation frequency. In the remainder of the paper, dimensionless quantities will be used. The accent in the symbols will be dropped and the symbols will refer to the dimensionless quantities.

\section{Flow visualizations}

In general, flow visualization is performed to obtain qualitative information on the flow pattern. Several techniques
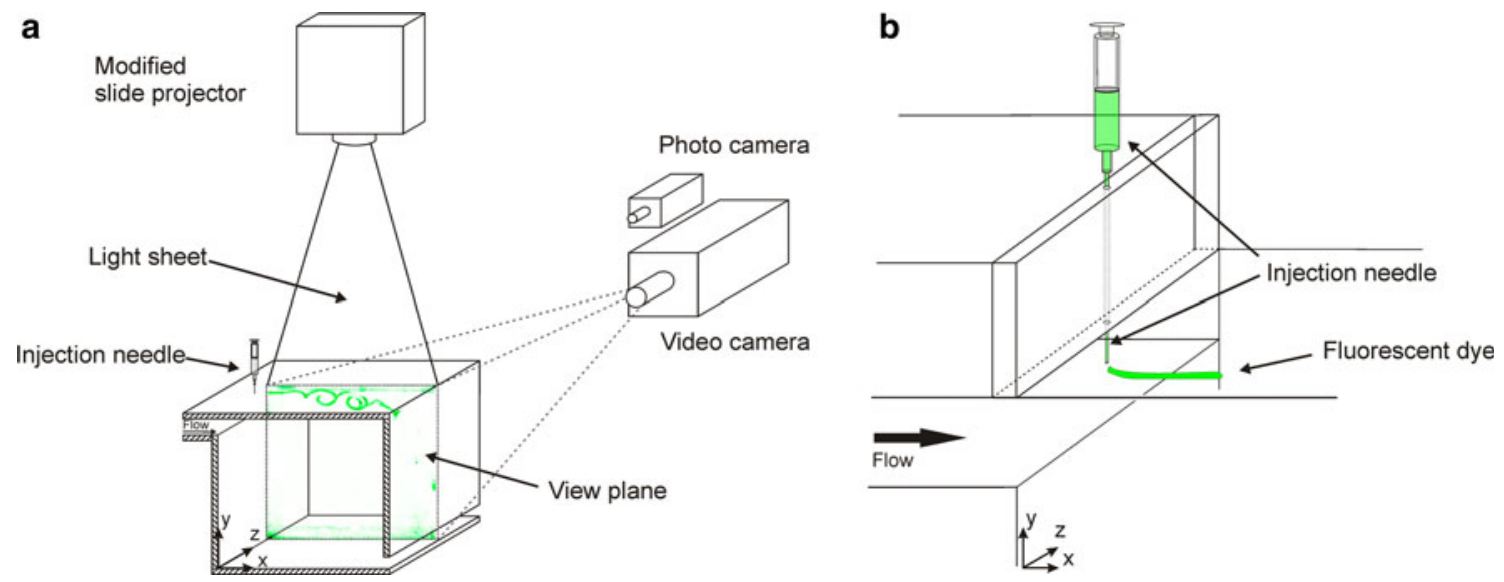

Fig. 2 a Overview of the reduced-scale setup during flow visualizations; b schematic representation of the injection of fluorescent dye are discussed in literature. Clayton and Massey (1967)among others-provide an overview of methods that can be used to perform flow visualizations in water. One of the described methods, the injection of streaks of dye, was used in this study to provide some first qualitative information on the flow regime. The flow visualizations were performed by illuminating a fluorescent liquid that was injected in the flow field (Fig. 2a). To obtain a fluorescent liquid, fluorescein was dissolved in water until saturation of the solution was reached. The saturated solution was inserted in the flow field in the vertical center plane $(z=0.5)$ in front of the inlet using an injection needle with an outer diameter of $0.6 \mathrm{~mm}$ and a length of $80 \mathrm{~mm}$ (Fig. 2b). The needle diameter was kept as small as possible to minimize the disturbing effect of the needle on the flow. To illuminate the fluorescent solution, a slide projector was installed above the test section, providing a light sheet in the center of the water cube with a width of about $0.01 \mathrm{~m}$, covering the entire test section of the cube (Fig. 2a). The flow pattern obtained by inserting and illuminating the fluorescent liquid was recorded with a video camera, and screen shots at a higher resolution were taken with a photo camera; both devices were positioned perpendicular to the flow direction as shown in Fig. 2a.

The flow visualizations were performed for $R e$ ranging from 300 to 3,700. Figure 3 shows instantaneous images of the flow inside the enclosure, taken in the vertical center plane $(z=0.5)$, for $R e \approx 1,000,1,750$ and 2,500. Figure $3 \mathrm{a}$ shows that instabilities are present for $R e \approx 1,000$. However, the presence of large-scale vortical structures cannot clearly be determined from the obtained instantaneous images. Clear large-scale vortical structures, however, can be distinguished in the outer region of the jet for the other two $R e$ numbers (Fig. 3b, c). These vortices are due to instability of the free shear layer that is situated in the outer region of the wall jet. The vortical structures are convected downstream where they break up. From the flow visualizations, it can be 

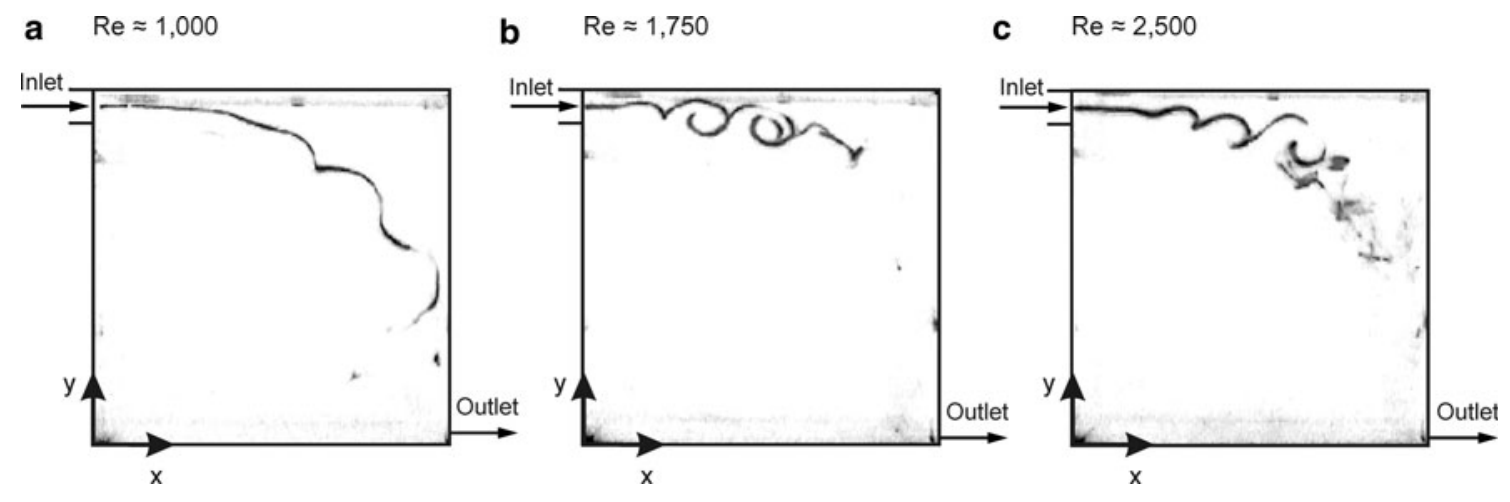

Fig. 3 Instantaneous images of the flow pattern inside the enclosure for $h=0.1$ and for $\operatorname{Re} \approx 1,000(\mathbf{a}), \operatorname{Re} \approx 1,750(\mathbf{b}), \operatorname{Re} \approx 2,500(\mathbf{c})$

concluded that transitional flow appears to be present for $R e$ up to at least 2,500. Furthermore, it can be concluded that the onset of jet instability occurs further upstream as $R e$ increases, which is in agreement with the findings of, among others, Kwon and Seo (2005). Note that this trend was observed from the unsteady evolution of the injected dye; this trend is not totally clear from the steady images depicted in Fig. 3. Based on the flow visualizations described in this section, PIV measurements were conducted for $R e$ ranging from 300 to 2,500, which is the range for which transitional flow is present.

\section{PIV measurement setup}

The PIV measurements in this study were conducted using a two-dimensional PIV system consisting of a Nd:Yag
$(532 \mathrm{~nm})$ double-cavity laser $(2 \times 200 \mathrm{~mJ}$, repetition rate $<10 \mathrm{~Hz}$ ) used to illuminate the field of view, and one CCD (charge-coupled device) camera $(1,376 \times 1,040$ pixel resolution, max. 10 frames/s) for image acquisition. The laser was mounted on a translation stage and was positioned above the cubic test section to create a laser sheet in the vertical center plane of the cube; the CCD camera was positioned perpendicular to the laser sheet plane (Fig. 4). Seeding of the water was provided by hollow glass microspheres ( $3 \mathrm{M}$; type $\mathrm{K} 1)$ with diameters in the range of $30-115 \mu \mathrm{m}$.

In order to determine the reliable time-averaged flow quantities, sufficient statistically-independent (uncorrelated) samples (i.e., PIV vector fields resulting from double image pairs) have to be acquired. Therefore, the PIV image acquisition frequency has to be sufficiently low. The required measuring frequency was estimated from the

Fig. 4 PIV measurement setup; the laser head is positioned above the test section using a translation stage. ROI1 indicates the region of interest $(L \times L)$ for the first measurement set, and ROI2 indicates the region of interest of $0.6 L \times 0.4 L(W \times H)$ for the second set

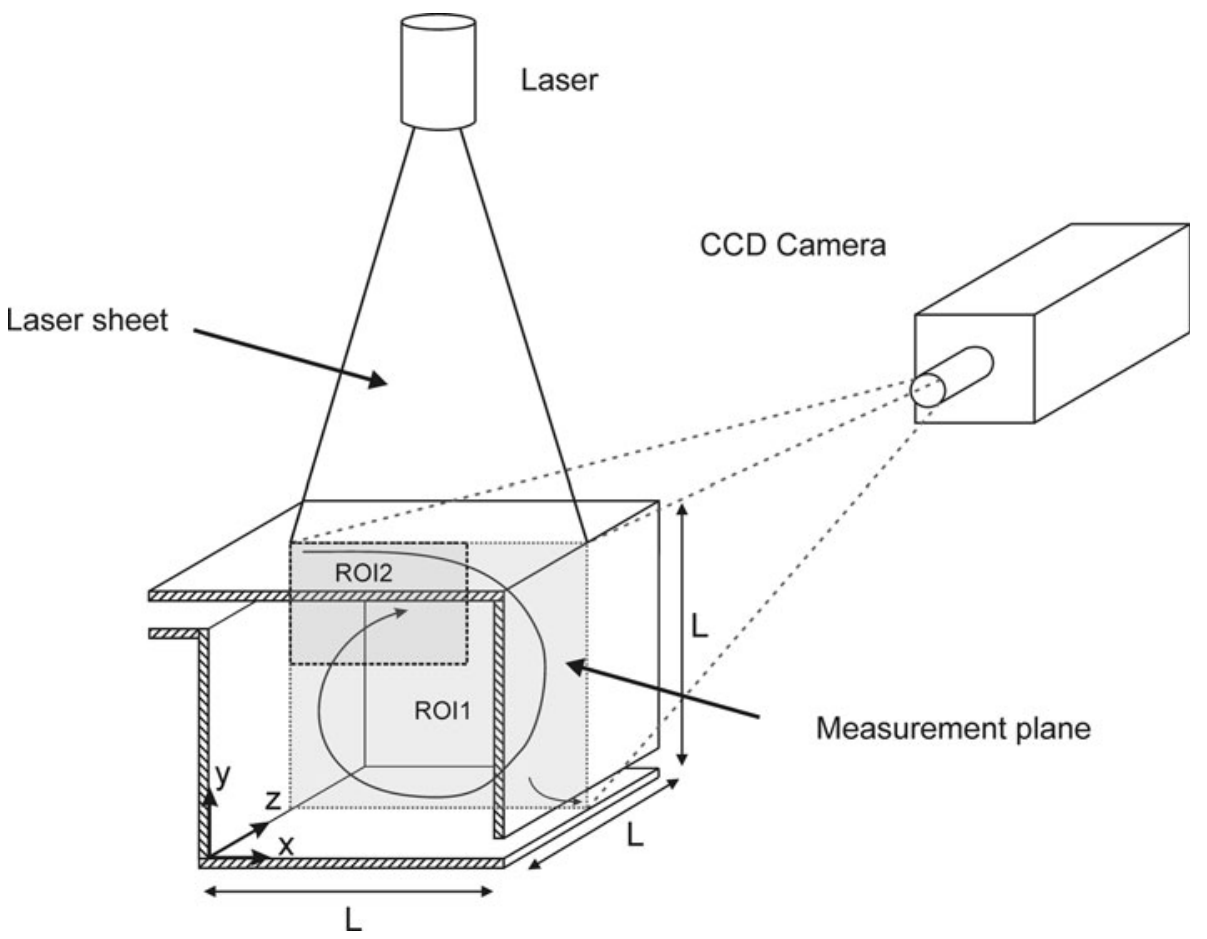


Table 1 PIV measurement parameters. The samples are double image pairs which result in instantaneous PIV vector fields

\begin{tabular}{lllllll}
\hline$R e[-]$ & 800 & 1,000 & 1,200 & 1,500 & 1,750 & 2,200 \\
Measuring frequency [Hz] & 1.34 & 1.67 & 2.00 & 2.00 & 2.00 & 2.00 \\
Number of samples [-] & 360 & 360 & 360 & 360 & 360 & 360 \\
Sampling time [s] & 270 & 216 & 180 & 180 & 180 & 3.00 \\
Frame rate $[\mu \mathrm{s}]$ & 40,000 & 40,000 & 35,000 & 25,000 & 20,000 & 180 \\
\hline
\end{tabular}

integral length scale (=inlet height) and the characteristic velocity (i.e., inlet velocity) and was set to the values shown in Table 1. Each measurement set consists of 360 uncorrelated samples. The errors associated with PIV measurements can be divided into systematic and repeatability errors. The systematic errors are present for each sample and consist of a range of errors that are associated with the PIV measurement technique and methodology (Prasad 2000). Prasad (2000) mentions 5 possible sources of errors (1) random error due to noise; (2) bias error; (3) gradient error; (4) tracking error; and (5) acceleration error. Due to the complexity of these errors, and the fact that a reduction in one error can lead to an increase in other errors, it is very difficult to provide a quantitative estimate of these errors on the measured velocity. To minimize the systematic errors, the best practice guidelines of Keane and Adrian (1990) and Prasad (2000) have been taken into account. The instantaneous PIV images were processed in two passes with decreasing interrogation areas, from $64 \times 64$ pixels to $32 \times 32$ pixels, both with a $75 \%$ overlap, to obtain instantaneous velocity vector fields. The vectors were only accepted when the ratio between the first and second tallest correlation peak for an interrogation area was above 1.3 to eliminate spurious vectors resulting from background noise or local insufficient seeding. The frame rate was chosen such that the particle displacement within an interrogation area between two frames was about 6 pixels, which satisfies the guidelines of Keane and Adrian (1990), who state that the particle displacement should be less than $1 / 4$ of the interrogation area size. The particle diameter on the images was about 2 pixels, which is in accordance with the particle diameter as discussed in Prasad (2000). The repeatability, or random, error is a statistical error. The uncertainty associated with the repeatability error can be assessed using the central limit theorem (e.g., Coleman and Steel 1999). The random uncertainty of the mean velocity $u_{r}$ can be calculated with

$u_{r}=\frac{1}{\sqrt{N}} \frac{z_{\alpha}}{2} \frac{r_{\mathrm{RMS}}}{R}$

with $N$ the number of samples (in this case $N=360$ ), $z_{\alpha} / 2$ a variable related to the chosen confidence interval, $r_{R M S}$ the root mean square of the measured two-dimensional velocity in a point and $R$ the mean two-dimensional velocity at the same location. For a confidence interval of $95 \%$, the corresponding value of $z_{\alpha} / 2=1.96$. The uncertainty of the measurement results is around $2-4 \%$ in the largest part of the test section and is slightly higher in the shear layer and boundary layer areas as a result of the locally higher turbulence levels. Note that $R$ is the local two-dimensional velocity and not the maximum jet velocity in this case.

The two-dimensionality of the flow was tested by performing measurements in two additional vertical planes at $z=0.417$ and $z=0.330$, which showed no significant differences in the time-averaged flow pattern between the three vertical planes. The time-averaged velocity fields were obtained by averaging the 360 instantaneous velocity vector fields.

Two sets of PIV measurements were performed in the vertical center plane $(z=0.5)$ of the water cube. The first set focuses on the entire cross-section of the cube, that is, a region of interest (ROI) of $L \times L$ (Fig. 4; ROI1). The second set focuses on a smaller region of interest of 0.6 $L \times 0.4 \mathrm{~L}(W \times H)$ in the proximity of the inlet, enabling a higher measurement resolution (Fig. 4; ROI2). The higher resolution provides more detailed information in this area with expected large velocity gradients. This information about the inlet conditions is important as boundary conditions for future numerical simulations.

\section{PIV measurement results}

In this section, we will present and discuss the results of the PIV measurements. The results for the case with $R e \approx 300$ are excluded because the observed flow pattern was very unstable, that is, the large recirculation region was not present in each instantaneous velocity vector field due to the very low momentum of the wall jet; therefore, averaging resulted in ambiguous results.

\subsection{Analysis of time-averaged results}

\subsubsection{Time-averaged velocity vector fields}

Figure 5 shows time-averaged velocity vector fields for $R e \approx 1,000,1,750$ and 2,500, for both sets of measurements. 
Fig. 5 Time-averaged velocity vectors for $\operatorname{Re} \approx 1,000(\mathbf{a}, \mathbf{b})$, $R e \approx 1,750(\mathbf{c}, \mathbf{d}), R e \approx 2,500$ $(\mathbf{e}, \mathbf{f}) .(\mathbf{a}, \mathbf{c}, \mathbf{e})$ Measurements for entire cross-section (ROI1); $(\mathbf{b}, \mathbf{d}, \mathbf{f})$ measurements with higher resolution near inlet (ROI2)
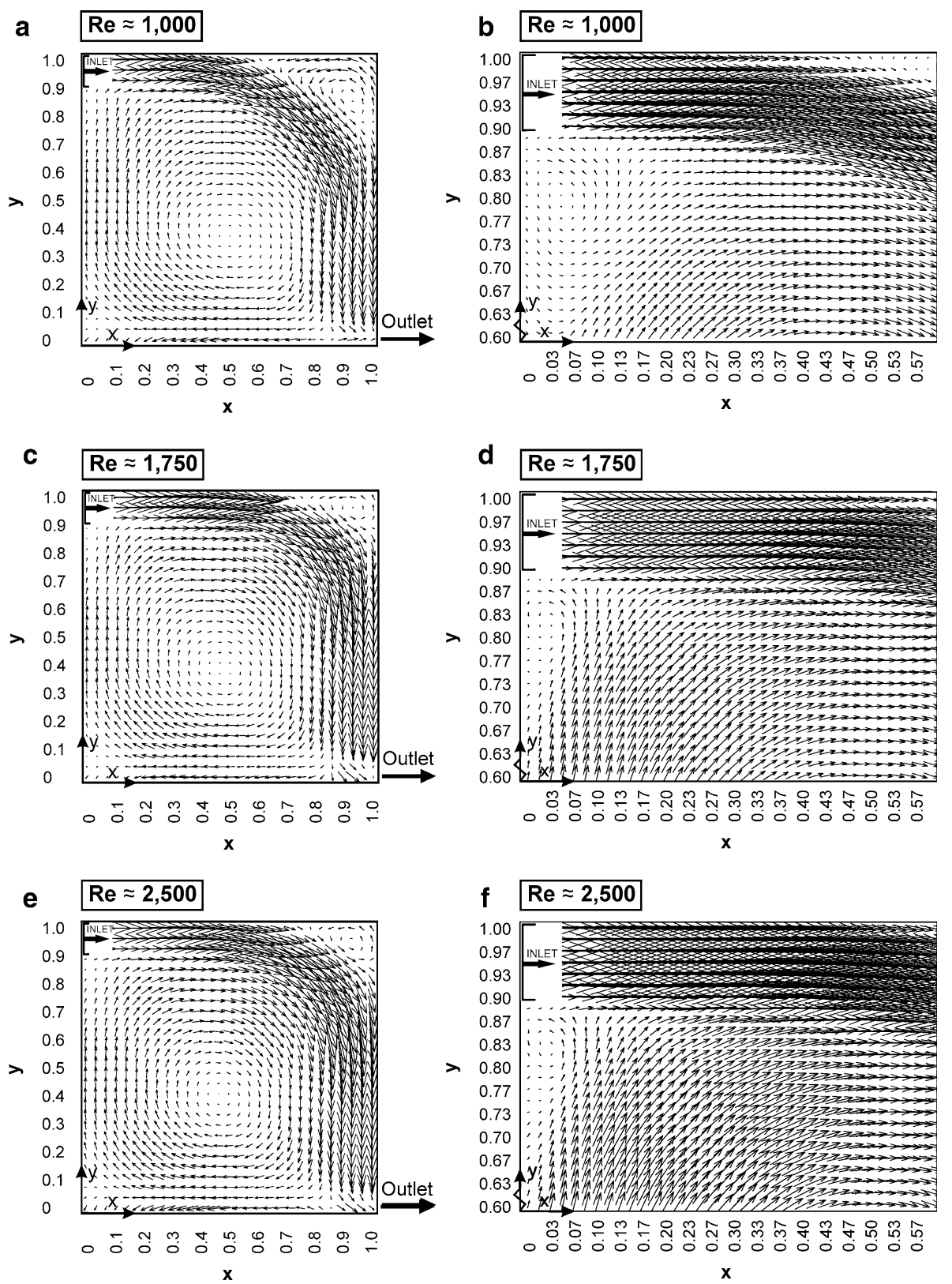

Note that only 1 out of 4 vectors is shown in all figures; that is, the actual resolution is sixteen times higher than shown. The velocity vectors are scaled with $R e$, and velocity vectors in the close vicinity of the inlet are omitted; due to reflections at the walls, the reliability of these vectors could not be guaranteed. Figure 5(a, c, e) show the large recirculation cell in the cube, which is driven by the wall jet. A smaller recirculation cell is present in the top right corner, and its size decreases with increasing $R e$. Figure $5(\mathrm{~b}, \mathrm{~d}, \mathrm{f})$ show the entrance of the wall jet and the presence of a very small recirculation cell just below the entrance. The size of this cell also decreases with increasing $R e$-values. Although the vector fields provide valuable information on the general flow pattern, for a more detailed analysis, profiles of time-averaged velocity, turbulence intensity and vorticity will be used.

\subsubsection{Time-averaged velocity and turbulence profiles near the inlet}

Figure 6 shows the profiles of dimensionless time-averaged $x$-velocity $U / U_{M}$ at distances $x=0.1,0.2,0.3,0.4$ and 0.5 , in the vertical center plane $(z=0.5)$ for $R e$ from 1,000 to 2,500. The coordinate system is given in Fig. 1. Figure 6a indicates that there are hardly any differences in the velocity profiles of the wall jet for the different Reynolds numbers at $x=0.1$. However, the enlarged graph in Fig. $6 \mathrm{f}$ and the $y_{C}$ values in Fig. 7 show that the distance $y_{C}$, 

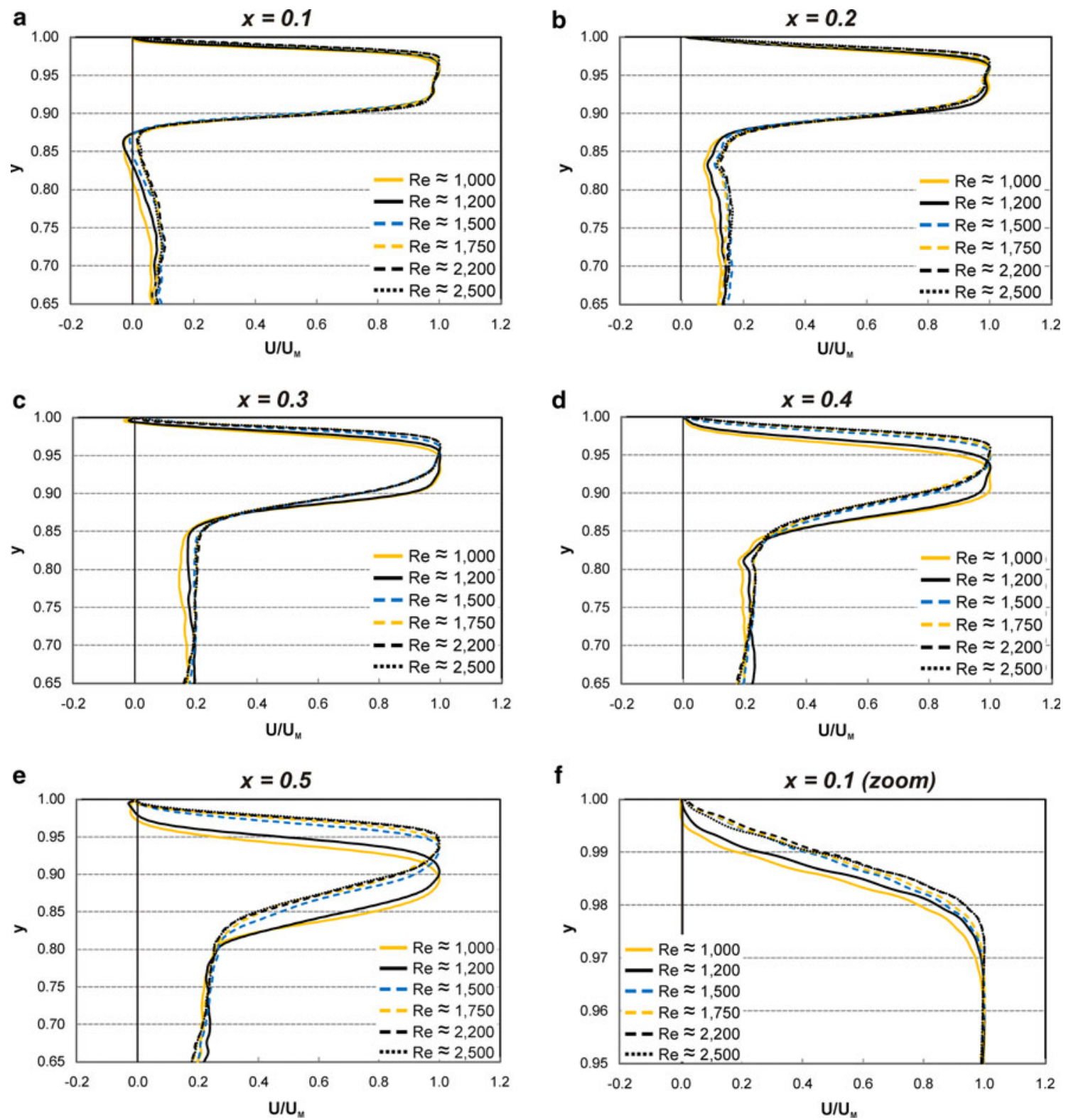

Fig. 6 Time-averaged profiles of $U / U_{M}$ at $x=0.1(\mathbf{a}), x=0.2(\mathbf{b}), x=0.3(\mathbf{c}), x=0.4(\mathbf{d}), x=0.5(\mathbf{e})$. $\mathbf{f}$ Presents an enlarged view of $U / U_{M}$ at $x=0.1$

measured from the bottom of the test section, increases slightly with increasing $R e$. Note that the small negative values at $x=0.1$ (Fig. 6a) for low $R e$-values are caused by the small recirculation cell below the jet, as depicted in Fig. 5(b, d, f). The profiles beneath the outer region of the wall jet $(y<0.87)$ also show a dependency on $R e$; higher $R e$-values result in larger $U / U_{M}$ values below the outer region (Fig. 6a). The velocity profiles further downstream show an increasing dependence of the local wall jet behavior on $R e$; the differences in $y_{C}$ for the six different $R e$-values increase with increasing $x$ (Fig. 7). Figure 6e, for example, shows that at $x=0.5$, the position of maximum jet velocity lies at $y_{C}=0.894$ for $R e \approx 1,000$, whereas $y_{C}=0.944$ for $R e \approx 2,500$. The negative values of $U / U_{M}$ in Fig. 6e indicate jet detachment from the top surface for the lower Reynolds numbers $(R e<1,500)$; jet detachment at $x=0.5$ is not yet present for higher $R e$, confirming that the location of jet detachment is Reynolds number dependent. Finally, Fig. 6(a,b) show a minimum value of $U / U_{M}$ below the outer region of the wall jet (at about $y=0.85$ ) for $x=0.1$ and $x=0.2$.

The profiles of $U / U_{M}$ are compared with the theoretical profiles of an unconfined wall jet to study the influence of the confinement on the wall jet profiles. Figure 8 shows the profiles of $U / U_{M}$ at five distances $(x=0.1,0.2,0.3,0.4$, $0.5)$, as well as the theoretical profiles for a laminar wall jet 


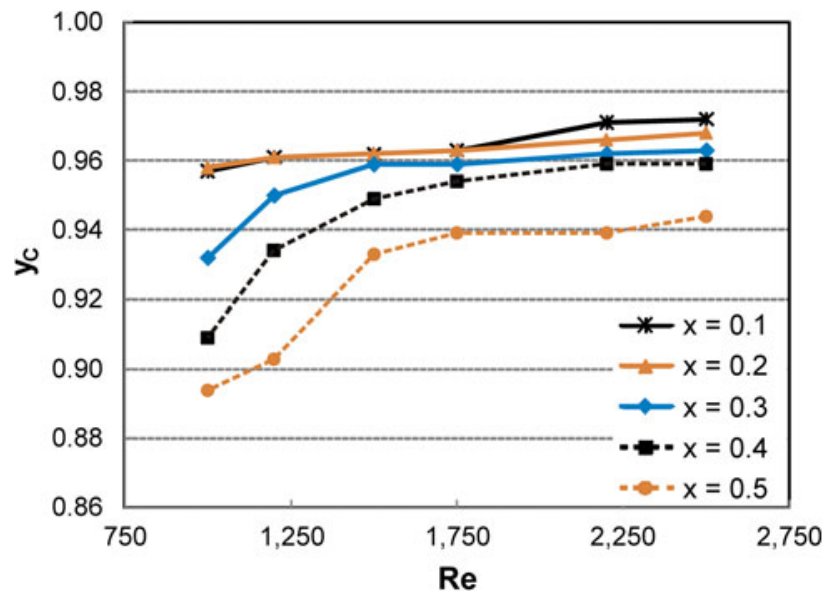

Fig. 7 Values of the position of maximum jet velocity $y_{C}$ obtained from the PIV measurements

(Glauert 1956) and for a turbulent wall jet (Verhoff 1963). Please note that the dimensionless $y$-coordinate is $y / y_{1 / 2}$ in this figure with $y / y_{1 / 2}=0$ at the top surface, instead of $y$ with $y=1$ at the top surface. The comparison for $R e \approx 1,000$ in Fig. 8a shows that the velocity profiles are in relatively good agreement with the theoretical profile for a laminar wall jet. Especially, the location of maximum velocity is in good agreement with the theory for a laminar wall jet $\left(y / y_{1 / 2} \approx 0.55\right)$, although the measured profiles have an almost top-hat profile instead of a parabolic profile. This different shape of the profile, especially at $x=0.1$ and $x=0.2$, is due to these measuring locations relatively close to the inlet, where the wall jet is not yet fully developed. At $x=0.5$, the wall jet has developed into a parabolic profile, however, the maximum velocity is now located at a larger distance from the top surface compared to the profile of Glauert (1956). This discrepancy can be explained by the separation of the wall jet at $x=0.5$ $\left(U / U_{M}<0\right)$, caused by an adverse pressure gradient as a result of the flow confinement (downstream wall). Figure $8 \mathrm{~b}$ compares the measured and the theoretical profiles for $R e \approx 1,750$. It can be seen that the measured profiles are shifting upwards compared to the profiles at $R e \approx 1,000$, as was also shown in Fig. 7. The profiles in the outer region of the wall jet resemble the theoretical profile of a laminar wall jet; however, in the inner region, the measured velocity profiles are now situated between the theoretical profiles for a laminar and a turbulent wall jet. The same holds for the location of maximum jet velocity $\left(U / U_{M}=1\right)$, which is situated between the theoretical values of a laminar wall jet $\left(y / y_{1 / 2} \approx 0.55\right)$ and of a turbulent wall jet $\left(y / y_{1 / 2} \approx 0.15\right)$. Finally, Fig. $8 \mathrm{c}$ shows the comparison for $R e \approx 2,500$, which is quite similar to the one for $R e \approx 1,750$. The measured profiles in the outer region resemble the theoretical profile for a laminar wall jet to a large extent, whereas those in the inner region are

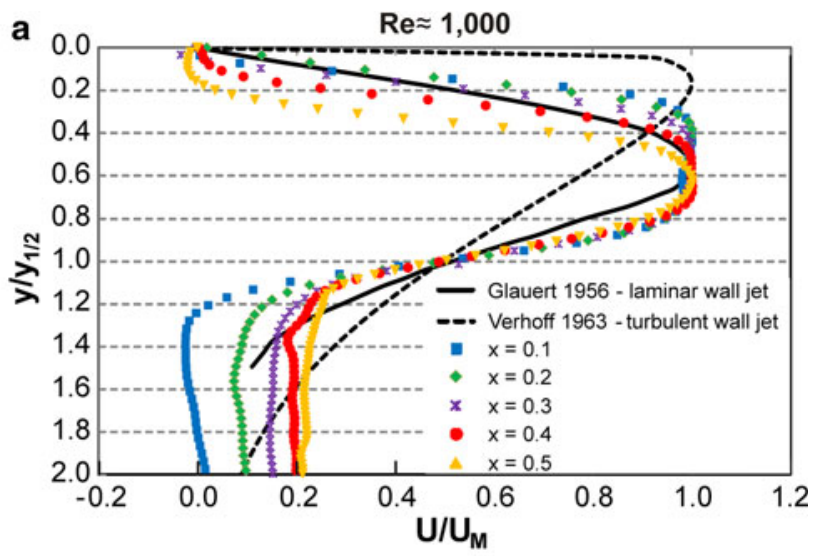

b
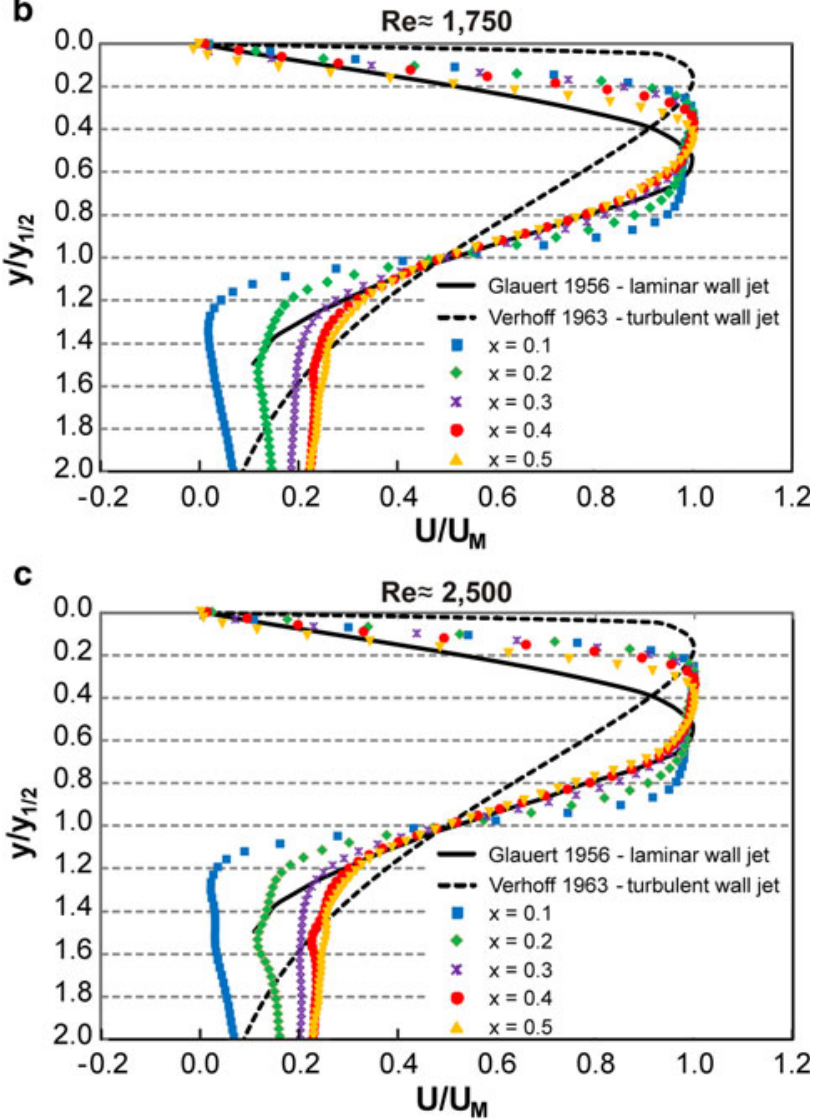

Fig. 8 Comparison between measured wall jet profiles and the theoretical profiles for a laminar wall jet (Glauert 1956) and a turbulent wall jet (Verhoff 1963) for $R e \approx 1,000(\mathbf{a}), R e \approx 1,750$ $(\mathbf{b}), \operatorname{Re} \approx 2,500(\mathbf{c})$

moving even further upwards to the top surface and consequently also to the theoretical profile of a turbulent wall jet. Again, the velocity profiles close to the inlet are more or less top-hat shaped, indicating that the wall jet is not yet fully developed at these locations.

The longitudinal turbulence intensities $u_{\mathrm{RMS}} / U_{M}$ at $x=0.1$ are shown in Fig. 9. The turbulence intensities in the core of the wall jet lie around 3\% and do not show a 


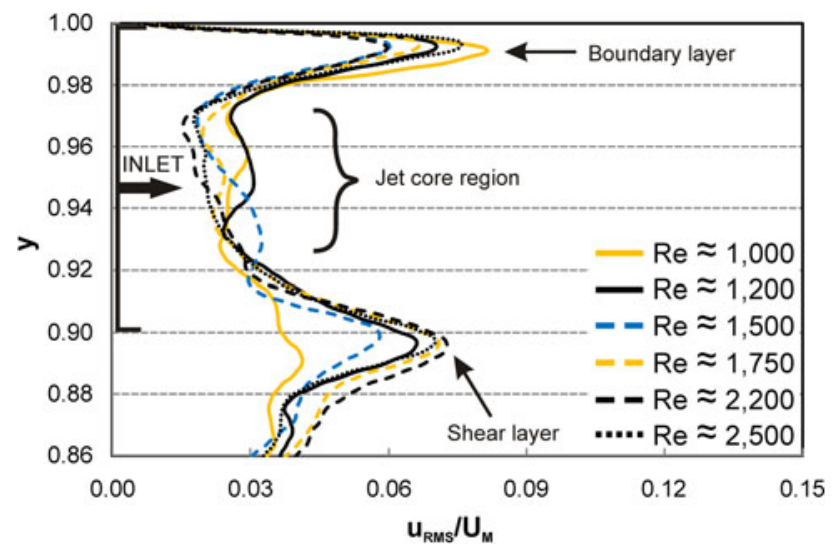

Fig. 9 Profiles of longitudinal turbulence intensity $\left(u_{\mathrm{RMS}} / U_{M}\right)$ at $x=0.1$

clear tendency with Re. The turbulence intensities near the edges of the wall jet are higher due to the large velocity gradients as a result of the boundary layer and shear layer flows in the inner and outer region, respectively. The turbulence intensities in the outer region vary with $R e$ : in general, an increase in $R e$ results in an increase in turbulence intensity, although the turbulence intensity for $R e \approx 1,500$ is lower than for $R e \approx 1,200$, and the turbulence intensity for $R e \approx 2,500$ is slightly lower than for $R e \approx 1,750$ and $R e \approx 2,200$. The general increase in turbulence intensity with increasing $R e$ in the shear layer is caused by a higher velocity gradient between the wall jet and the recirculation zone, resulting in a higher turbulence level in this region. For the inner region, no clear tendency can be distinguished; the highest values are present for $R e \approx 1,000$, while for $R e \approx 1,500$ and $R e \approx 2,200$, the lowest turbulence intensities are present. Therefore, it is not possible to draw any firm conclusions on the $R e$-dependency of the turbulence intensity in the inner region.

\subsubsection{Time-averaged velocity profiles in the entire flow domain}

The wall jet enters the confined space and forms a large recirculation zone that fills the cubical flow domain (Figs. 1a, 5a, c, e). Figure 10a shows profiles of the timeaveraged non-dimensional $x$-velocity $U / U_{M}$, taken at $x=0.2$ for $R e$ from 800 to 2,500. The profiles for different $R e$ are almost identical in the near-wall region $(y>0.9)$, as also illustrated in Fig. 6b. In the outer region of the wall jet, the velocity decreases to values around $U / U_{M}=0.05$. Locally, in the region $0.8<y<0.9$, a lower velocity is observed. Below this area of lower velocity, which was also depicted in Fig. $6 \mathrm{~b}, U / U_{M}$ shows an almost linear decrease to values around $U / U_{M}=-0.2$ at $y=0.05$. The profiles of $U / U_{M}$ are almost identical in the region from $y=0.6$ to $y=0.2$. Note that the profiles are not shown for $y<0.05$. The results in this part of the cube are inaccurate due to reflections of the laser sheet on the glass bottom of the cube. In addition, note that small differences are present between the velocity profiles in Fig. 10a and in Fig. 6b $(x=0.2)$ below the wall jet $(0.8<y<0.9)$. In this area, the velocities obtained from the measurements in ROI1 (Fig. 10a) are in general slightly lower than those obtained from ROI2 (Fig. 6b). The reason for this small discrepancy is the inability to accurately capture the small-scale recirculation zone just below the entrance of the wall jet due to the lower measuring resolution for ROI1. This recirculation zone directs the vertical flow along the upstream wall in the streamwise $(x)$ direction, and as a result, the horizontal velocity component in this area increases. This flow feature is also clearly visible in Fig. 5(b, d, f) and briefly addressed in Sect. 5.1.1. However, the small recirculation cell below the entrance of the jet is not accurately detected in ROI1 (see Fig. 5a, c, e). As a result, the vertical flow along the upstream wall remains mainly vertical until it reaches the
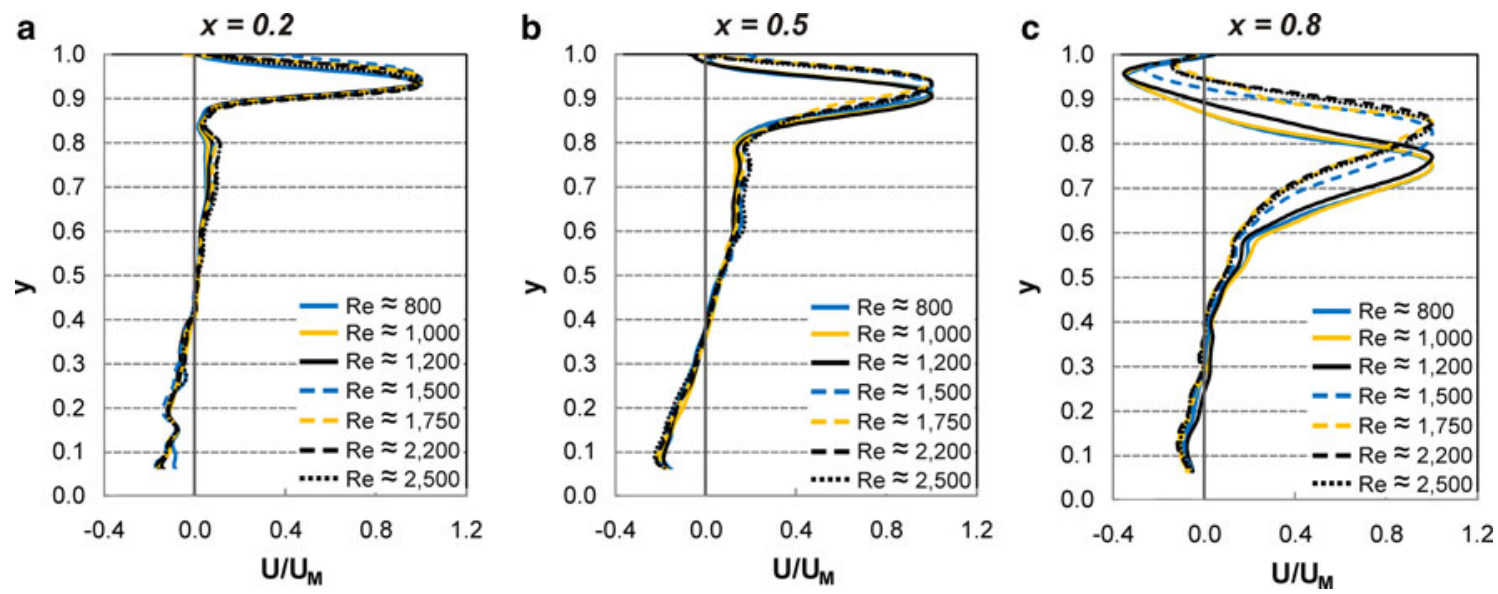

Fig. 10 Time-averaged profiles of $U / U_{M}$ for $x=0.2(\mathbf{a}), x=0.5(\mathbf{b}), x=0.8(\mathbf{c})$ 
wall jet; the $x$-velocity remains relatively small, whereas the $y$-velocity is larger compared to the one measured at ROI2.

Profiles of $U / U_{M}$ at $x=0.5$ are shown in Fig. 10b. As already shown in Fig. $6 \mathrm{e}$, the profiles at $x=0.5$ show $R e$-dependency in the wall jet region. The value of $y_{C}$ is lower for lower $R e$-values. From $y=0.6$ to $y=0.1, U / U_{M}$ decreases almost linearly from 0.15 to -0.2 ; again, no clear $R e$-dependency is present in this area. The height of the center of the recirculation zone, $U / U_{M}=0$, lies approximately at $y=0.4$ for all $R e$-values.

Figure 10c shows $U / U_{M}$ at $x=0.8$. Clear differences can be seen in the profiles for different $R e$. The negative values for $U / U_{M}$ in the inner region indicate detachment of the wall jet boundary layer, resulting in a small recirculation cell downstream of the detachment point. The size of this recirculation cell, and thus the area with negative values of $U / U_{M}$, increases with decreasing $R e$. The vertical location of the maximum velocity depends strongly on $R e$, for example, for $R e \approx 1,000, y_{C}=0.754$, whereas for $R e \approx 2,500, y_{C}=0.843$. Below the wall jet region, $y<0.6, R e$-dependency is still present, although the differences are far less pronounced.

\subsubsection{Time-averaged vorticity profiles}

Figure 11 shows profiles of $z$-vorticity $\omega_{z}$ at $x=0.2$ and $x=0.5$ for seven different $R e$-values. The positive and negative $\omega_{z}$-values above and below $y=0.94$ (Fig. 11a) clearly indicate the upper and lower parts of the jet, with $\mathrm{d} U / \mathrm{d} y<0$ and $\mathrm{d} U / \mathrm{d} y>0$, respectively (compare with Fig. 10a, b). Note that $U / U_{M}$ has the highest value at $y=0.94$; at this location, $\omega_{z}=0$, indicating the interface between positive and negative vorticity. The very weak negative vorticity in the range $0.1<y<0.8$ is directly connected with the large clockwise-rotating recirculation cell. Figure 11a shows that there is no clear dependency of $\omega_{z}$ on $R e$; below $y=0.94$, the values of $\omega_{z}$ are clearly $R e$-independent, whereas above $y=0.94$, the values of $\omega_{z}$ do not overlap, but also do not show a trend with $R e$. The vorticity distribution at $x=0.5$ is depicted in Fig. $11 \mathrm{~b}$ and shows roughly the same features as at $x=0.2$. The border between the negative vorticity, induced by the shear layer, and the positive vorticity as a result of the boundary layer lies at $y=0.91$ for $R e$-values until 1,200 and around $y=0.93$ for $\operatorname{Re}>1,200$. From $y=0.75$ until $y=0.1$, a low and almost constant negative vorticity is present, which is associated with the clockwise-rotating recirculation cell extending over the lower part of the enclosure. Apparently, the motion in this cell is close to a rigid-body rotation. The differences in $\omega_{z}$ for the different $R e$-values are more pronounced at $x=0.5$ (Fig. 11b) than at $x=0.2$. At $x=0.5$, the locations of maximum $\omega_{z}$ differ for different values of $R e$, which is consistent with the $R e$-dependent profiles of $U / U_{M}$ at this location, as indicated in Fig. 10b. Finally, the values of $\omega_{z}$ in the jet region and below the jet region do not show a clear trend with $R e$.

\subsection{Analysis of instantaneous flow field}

\subsubsection{Instantaneous velocity vector fields}

The transient flow features are studied using instantaneous velocity vector fields. Figure 12 shows instantaneous velocity vector fields for $R e \approx 1,000,1,750$ and 2,500, at two different positions in time. Note that only 1 out of 2 vectors is shown; that is, the actual resolution is four times higher than shown. The velocity vectors are scaled with $R e$ and the (mostly spurious) velocity vectors in the close vicinity of the inlet are not shown. A KelvinHelmholtz-type instability of the wall jet is clearly observed. Figure 13(a, c, e) show instantaneous vector
Fig. 11 Time-averaged profiles of dimensionless $z$-vorticity $\omega_{z}$ at $x=0.2(\mathbf{a}), x=0.5(\mathbf{b})$
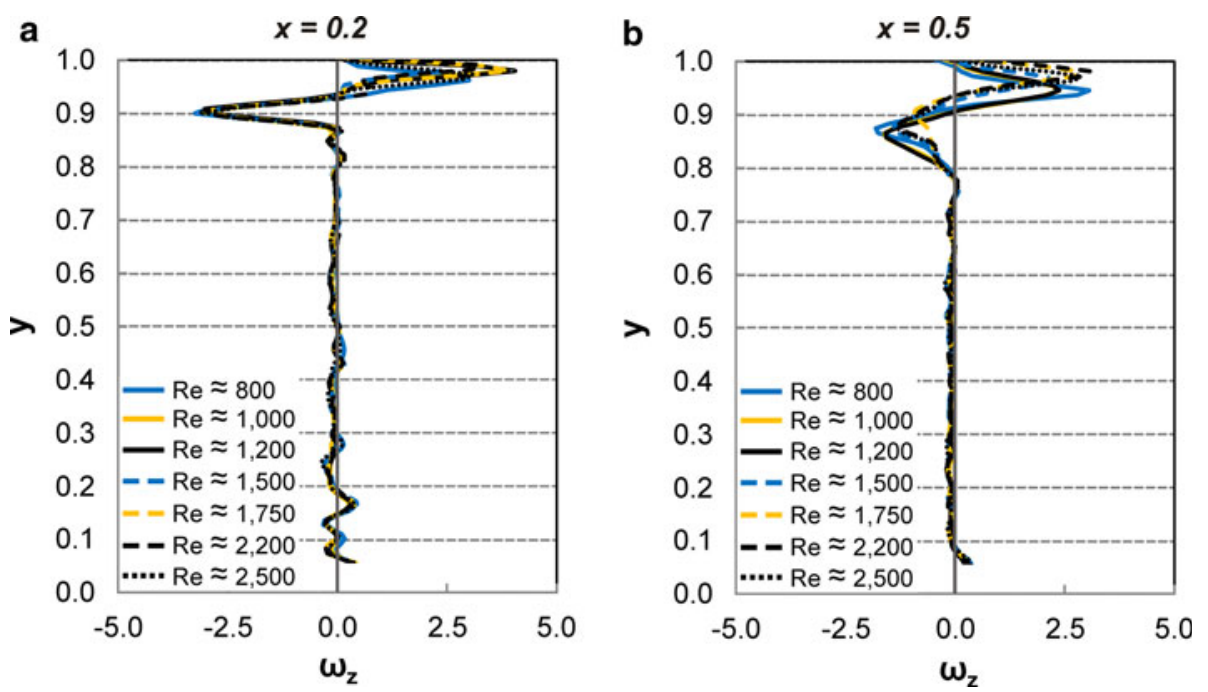

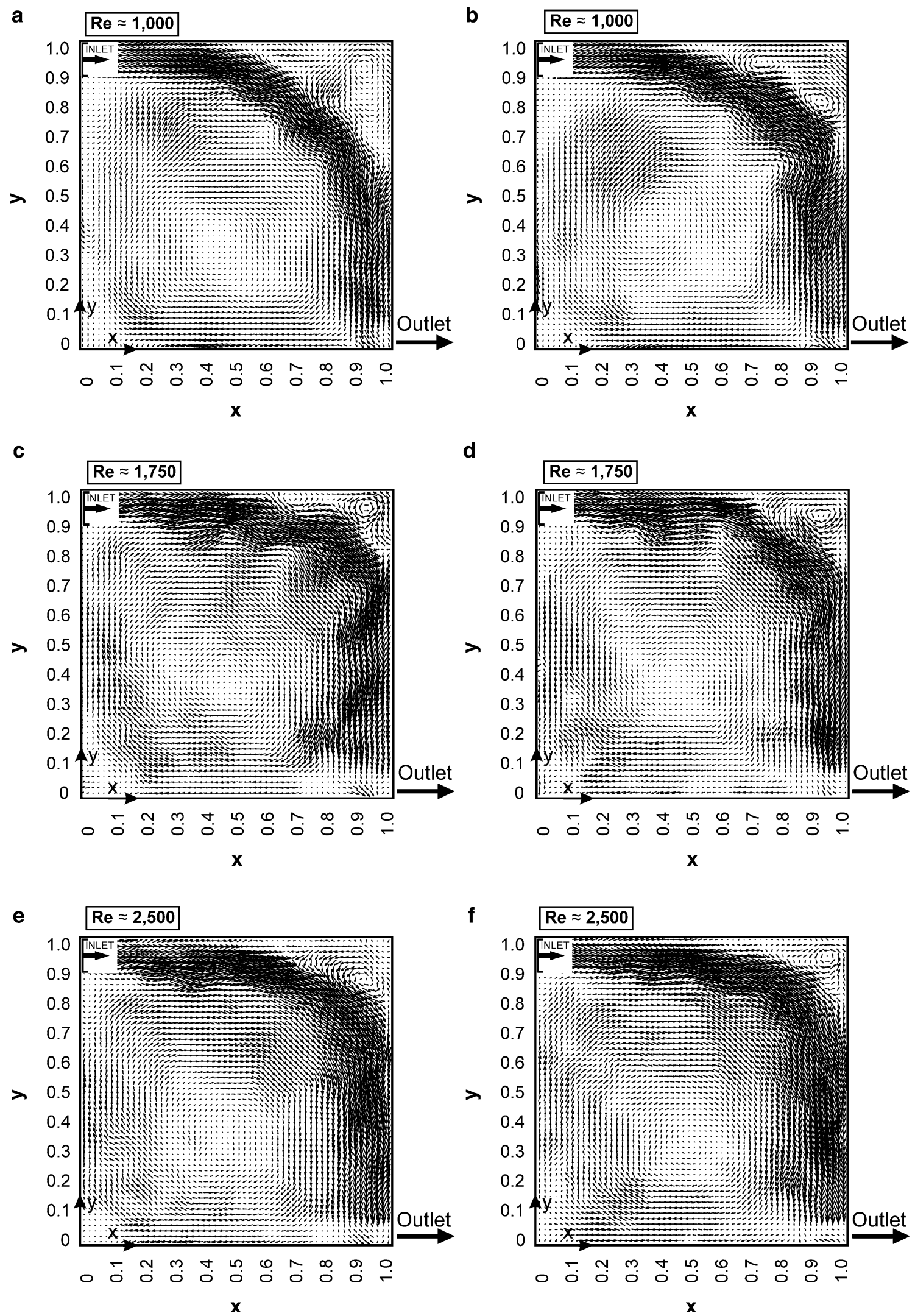

Fig. 12 Instantaneous velocity vector fields at two different positions in time for $\operatorname{Re} \approx 1,000(\mathbf{a}, \mathbf{b}) ; \operatorname{Re} \approx 1,750(\mathbf{c}, \mathbf{d}) ; \operatorname{Re} \approx 2,500(\mathbf{e}, \mathbf{f})$ 


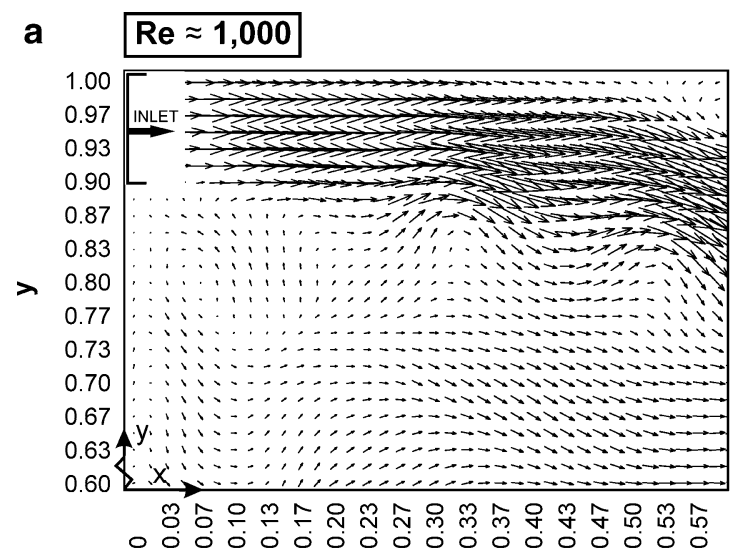

$\mathbf{x}$

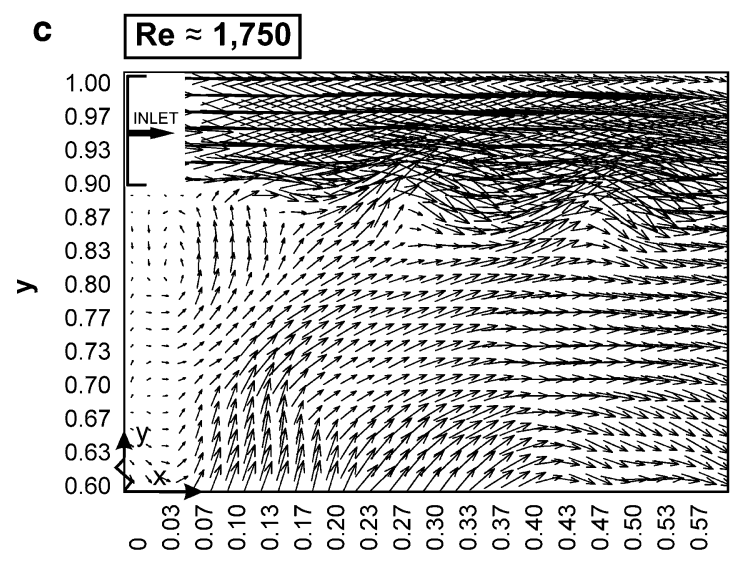

$\mathbf{x}$

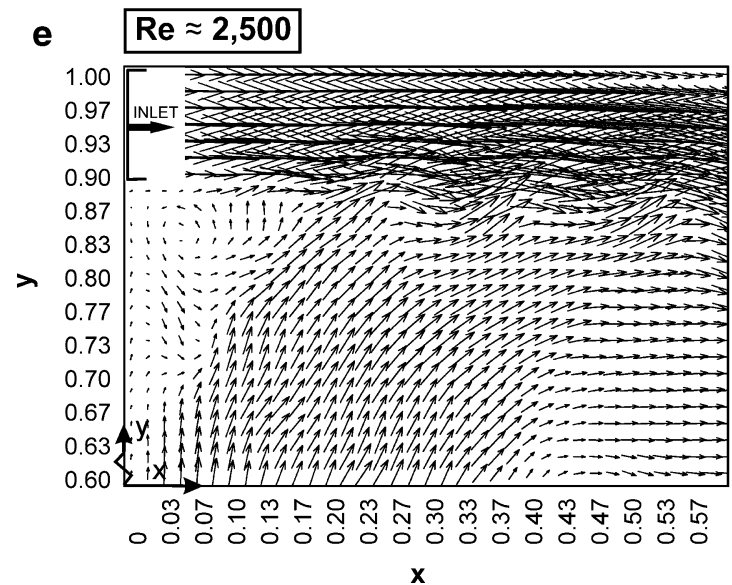

Fig. 13 Instantaneous velocity vector fields. Absolute velocity vectors are depicted in panels $(\mathbf{a}, \mathbf{c}, \mathbf{e})$, whereas $(\mathbf{b}, \mathbf{d}, \mathbf{f})$ show the vector fields obtained by subtracting the area-averaged instantaneous

fields for the same $R e$-values from the second measurement set with a reduced region of interest (ROI2). In this figure, only 1 out of 4 vectors is shown; that is, the actual resolution is sixteen times higher than shown, and the velocity vectors are again scaled with $R e$. Kelvin-Helmholtz-type instability waves are again visible at the outer
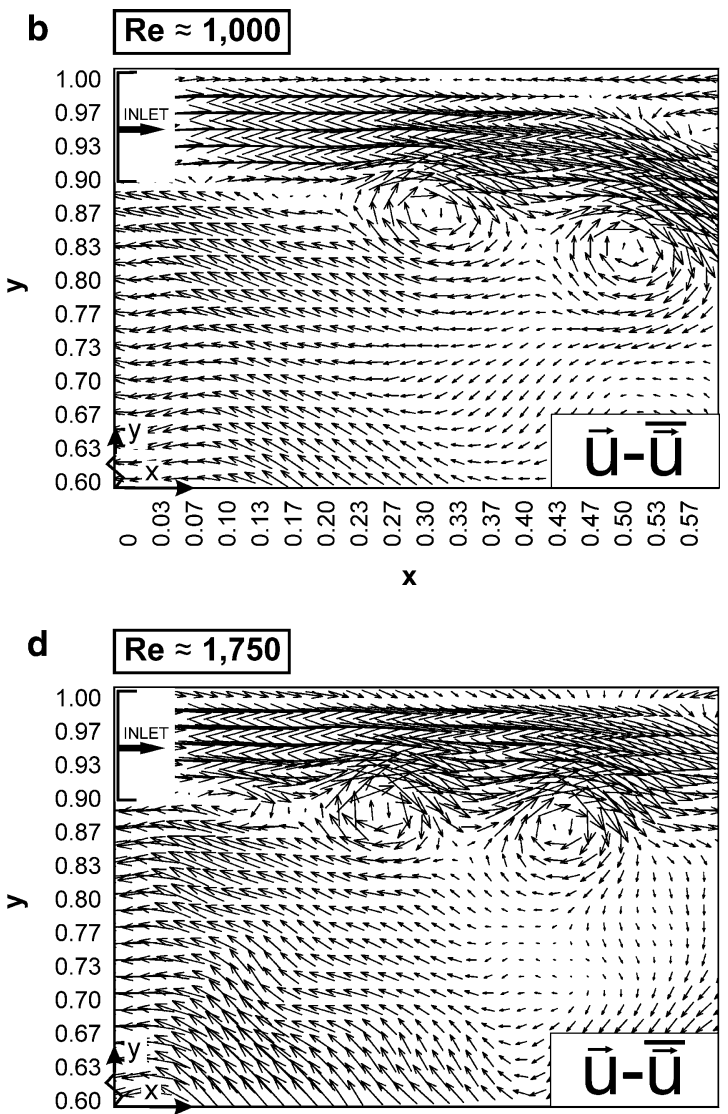

。ํ.

$\mathbf{x}$

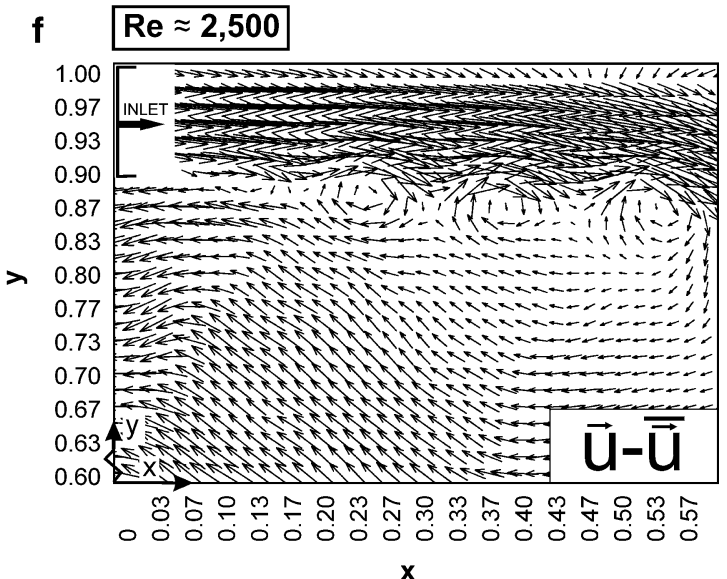

velocity from the absolute instantaneous velocities. $R e \approx 1,000$ $(\mathbf{a}, \mathbf{b}), \operatorname{Re} \approx 1,750(\mathbf{c}, \mathbf{d}), \operatorname{Re} \approx 2,500(\mathbf{e}, \mathbf{f})$

region of the wall jet, which will grow and form the onset to the formation of discrete vortical structures. The presence of vortical structures is clearly visible in Fig. 13(b, d, f), which are obtained by subtracting the area-averaged instantaneous velocity from the absolute instantaneous velocities. 


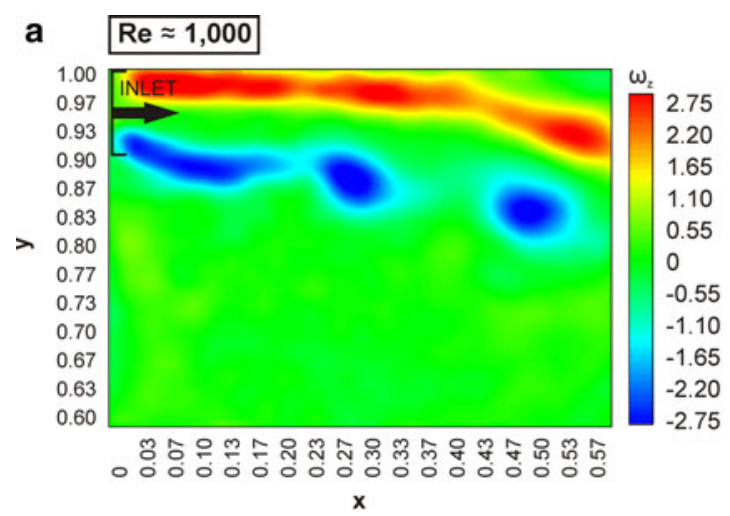

C $\operatorname{Re} \approx 1,750$
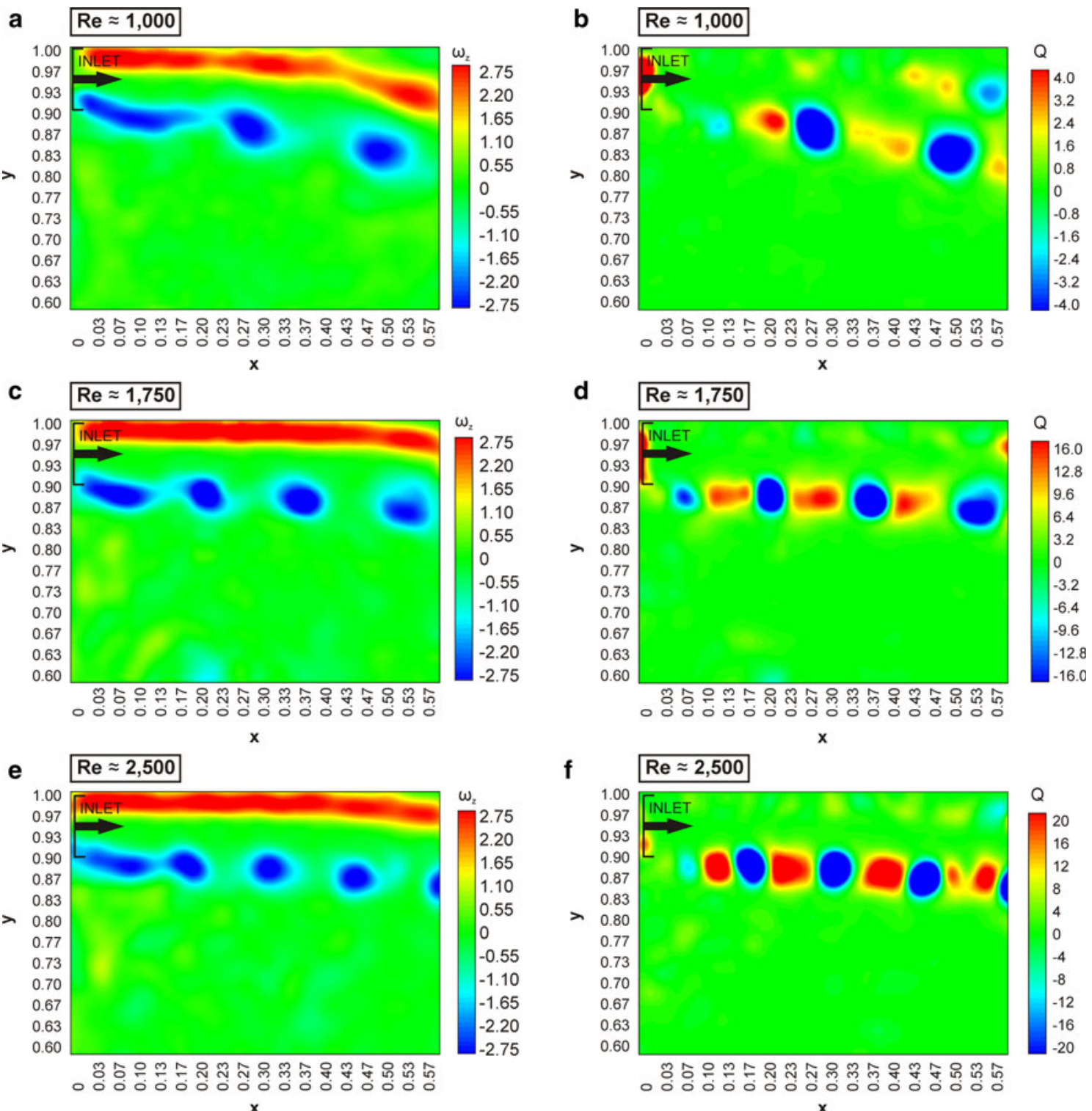

Fig. 14 Contour plots of the vorticity $\omega_{z}$ and the corresponding contour plots of the Okubo-Weiss function $Q$ for $R e \approx 1,000(\mathbf{a}, \mathbf{b})$, $R e \approx 1,750(\mathbf{c}, \mathbf{d}), R e \approx 2,500(\mathbf{e}, \mathbf{f})$

\subsubsection{Application of $Q$ criterion}

A further analysis of the vortical structures in the flow domain is conducted using the Okubo-Weiss function, which was independently defined by Okubo (1970) and Weiss (1991). Based on the Okubo-Weiss function, the $Q$ criterion for the center plane $(z=0.5)$ can be defined as follows:

$$
\begin{aligned}
Q(x, y, z & =0.5) \\
& =\left(\frac{\partial u}{\partial x}-\frac{\partial v}{\partial y}\right)^{2}+\left(\frac{\partial u}{\partial y}+\frac{\partial v}{\partial x}\right)^{2}-\left(\frac{\partial v}{\partial x}-\frac{\partial u}{\partial y}\right)^{2}
\end{aligned}
$$

The first two terms denote the normal strain component and shear strain component, respectively, and the last term is associated with the vorticity $\omega_{z}$. Values of $Q>0$ indicate strain dominated, hyperbolic, flow regions, whereas $Q<0$ indicates rotation-dominated regions (elliptic flow regions). The Okubo-Weiss function is valid for two-dimensional flow and has been successfully used by, among others, Vosbeek et al. (1997), Isern-Fontanet et al. (2004), Molenaar et al. (2004) and Cieślik et al. (2010). In this study, $Q$ is determined for the center $x, y$-plane $(z=0.5)$.

Figure 14 shows $\omega_{z}$ contours and $Q$-contours for instantaneous velocity fields for $R e \approx 1,000,1,750$ and 2,500 , respectively. Figure $14(\mathrm{a}, \mathrm{c}, \mathrm{e})$ show the vorticity 
distribution for the three values of $R e$, clearly illustrating the presence of vorticity in the inner and outer sides of the jet, associated with the boundary layer and the free shear layer, respectively. Figure 14(b, d, f) show the contours of $Q$, illustrating the presence of a vortex train in the outer region of the wall jet as a result of Kelvin-Helmholtz-type instability (shear layer). In the vortex train, one can distinguish rotation-dominated regions $(Q<0)$ alternating with strain-dominated regions $(Q>0)$. Figure 14(a, c, e) show that between the vortices, $\omega_{z} \approx 0$; due to the strain in these regions, the resulting value of $Q$ becomes positive (hyperbolic flow region) as depicted in Fig. 14(b, d, f). In contrast to the positive and negative $Q$-values in the outer region, the $Q$-values in the inner region lie around $Q \approx 0$. In good approximation, the flow in the wall jet boundary layer is characterized by the absence of a vertical velocity component $(v=0)$ and a negligibly small horizontal velocity gradient $\partial \mathrm{u} / \partial x$, which indeed confirms that $Q \approx 0$ in the inner region. However, Fig. 14b shows that for $R e \approx 1,000$, a vortical structure is present in the inner region at $x=0.55(Q<0)$, which is a location downstream of the point of jet detachment (for $R e \approx 1,000$ : $0.4<x<0.5)$. This observation corresponds to those of Bajura and Catalano (1975) and Gogineni and Shih (1997) that the development of vortices in the outer region is followed by the development of vortices in the inner region of the wall jet, which occurs after detachment of the boundary layer from the wall due to an adverse pressure gradient. Furthermore, it can be concluded that there is an increase in the number of vortical structures per unit length for increasing $R e$-values, indicating an increased vortex formation frequency with increasing $R e$. As a result, the distance between the two consecutive vortical structures decreases with increasing $R e$.

The instantaneous velocity fields in combination with the knowledge on the presence of vortical structures in the outer region of the wall jet can be used to analyze the instantaneous velocity profiles of the wall jet. Figure 15 again shows the vortical structures in the outer region of the wall jet for $R e \approx 1,000$. The dashed lines indicate the centers of the vortical structures in the outer region of the wall jet, whereas the solid line indicates a position between the two vortical structures. Figure $15 \mathrm{~b}$ shows the instantaneous velocity profiles taken at the two dashed lines and the solid line. The minimum instantaneous $x$-velocity $u / u_{M}$ in the outer region of the wall jet is 0.208 at the line between the two vortical cores, while the minimum $u / u_{M}$ is 0.022 and 0.066 at the lines through vortex core 1 and vortex core 2 , respectively. The presence of the clockwiserotating vortical structures results in lower instantaneous values of $u / u_{M}$ in the outer region of the wall jet, to be more precise, at the location where the lower edges of the vortical structures are present.

\subsubsection{Analysis of Strouhal number}

Since a clear periodicity of the flow was observed, the relation between $R e$ number and Strouhal number, defined as $S t=(f h) / U_{M}$, with $f$ the vortex formation frequency, $h$ the inlet height and $U_{M}$ the maximum $x$-velocity at $x=0.1$, is analyzed in this section. This relation has been the subject of several studies in the past. Namer and Ötügen (1988), among others, analyzed the relationship between $R e$ and $S t$ for free plane jets and $R e$ ranging from 1,000 to 7,000 . They stated that in their experiments, $S t$ was independent of the $\operatorname{Re}(S t \approx 0.273)$, even for $R e=1,000$, which is expected to result in transitional flow. However, Suresh et al. (2008) did find a dependency of $S t$ on $R e$ for free plane jets, at least for $R e<2,000$. For higher $R e$, they found that $S t$ reached an asymptotic value of around 0.36. It should be noted that all studies described above were performed for free plane jets in an unconfined space.

The vortex formation frequency in this study was determined based on the average distance between two adjacent vortical structures and the local maximum jet velocity $U_{M}$. Other studies reported vortex formation frequencies based on Fast Fourier Transformations (FFT); however, for the vast majority of the measurements in this study; the measuring frequency was too low (sampling frequency $<$ Nyquist frequency) to be able to determine $S t$ using a frequency spectrum. Only for $R e \approx 1,000$ and $R e \approx 1,200$, the measuring frequency was high enough to apply FFT and to determine the vortex formation frequency. Comparison of FFT with the used method showed a good agreement between results from both methods $( \pm 5 \%)$.

Figure 16 shows the calculated $S t$-values as a function of $R e$. The $S t$-values clearly increase with increasing $R e$. The presented results partly agree with the findings of Suresh et al. (2008) that St increases with Re. However, in their case, $S t$ reached an asymptotic value at $R e=2,000$, whereas no sign of an asymptotic value can be seen in Fig. 16 yet. A possible explanation might be the fact that the experiments in this study are all well within the transitional range and are consequently $R e$-dependent, whereas the onset to turbulent flow might have occurred at lower $R e$-values in the experiments by Suresh et al. (2008). Furthermore, their experiments were performed for a free jet and not for a wall jet and in air instead of water. Namer and Ötügen (1988) stated that the St-value, based on the vortex formation frequency, is constant. Our results, however, clearly show an increase in $S t$ with increasing $R e$ (Fig. 16), in line with the findings of Suresh et al. (2008). Again, possible explanations might be the flow regime during the measurements (turbulent vs. transitional), and the fact that they studied free plane jets instead of confined 

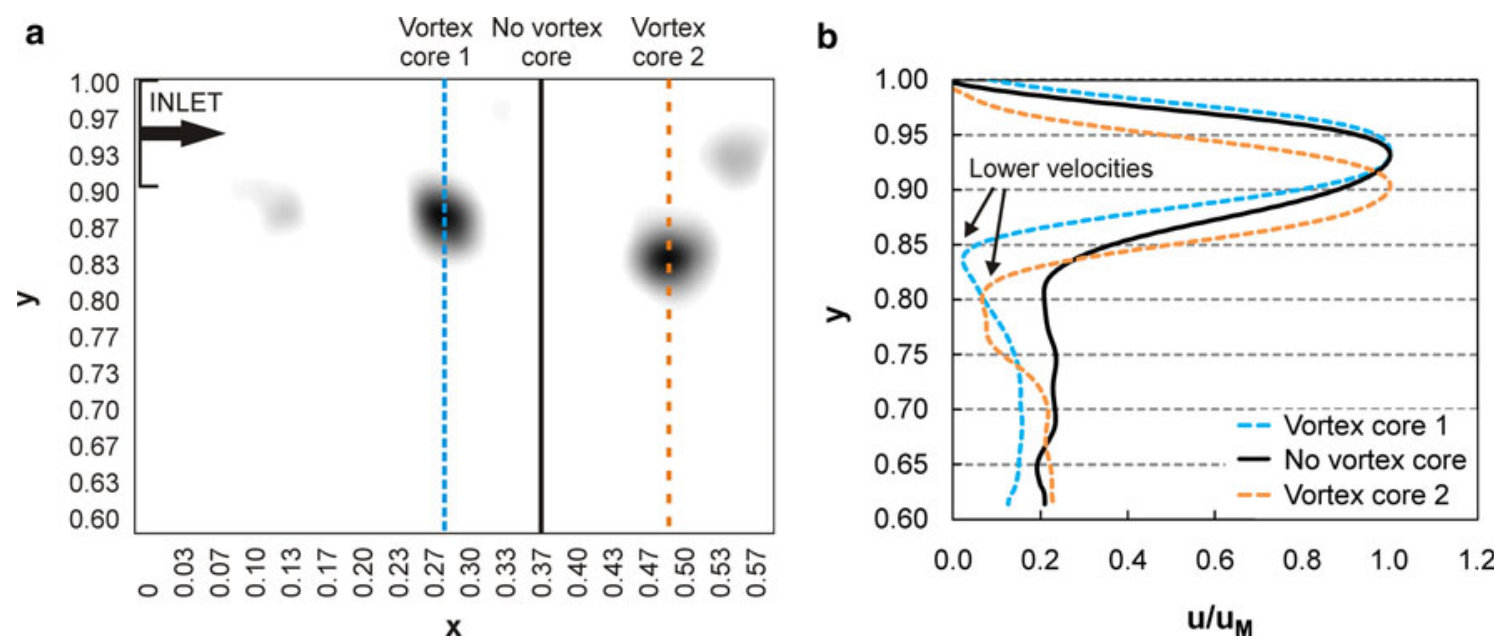

Fig. 15 a Contours of $Q<0$ for $R e \approx 1,000$. b Corresponding instantaneous velocity profiles $\left(u / u_{M}\right)$ at the dashed lines and the solid line, illustrating the influence of the clockwise-rotating vortical structures on the instantaneous values of the $x$-velocity $\left(u / u_{M}\right)$

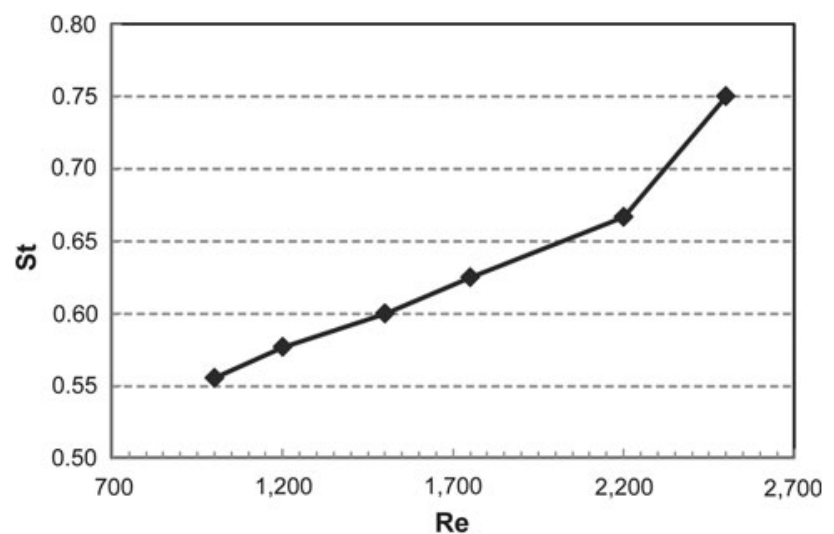

Fig. 16 Observed relationship between Strouhal number St and Reynolds number $R e$

plane wall jets. Furthermore, their study was conducted for an air jet.

\section{Discussion}

In this paper, the experimental results of a study on a transitional plane wall jet in a confined space have been presented and discussed. The experimental work consists of flow visualizations and PIV measurements to analyze the flow pattern. PIV measurements were performed for Reynolds numbers from 300 to 2,500, since the flow visualizations have shown that transitional flow is present for at least this range of $R e$-values.

In accordance with previous studies on transitional jets, the present study has shown that jet properties, such as time-averaged velocity, turbulence intensity and Strouhal number, show a $R e$-dependency. Unfortunately, a detailed comparison with previously published experimental data was not possible, since, to the knowledge of the authors, papers in which transitional plane wall jets in a confined space are analyzed using high-resolution measurements have not been published so far. However, a comparison with theoretical profiles was performed and showed that in general the measured wall jet profiles were situated between the theoretical profiles for a laminar and a turbulent wall jet. Furthermore, the comparison illustrated the effect of the flow confinement.

In Sect. 1, a short description was given of the five stages that generally occur during the transition from laminar to turbulent flow in jets, as identified by Bajura and Catalano (1975) based on flow visualizations and hot-film anemometry. In the present study, the presence of the following four stages can be identified: (1) formation of discrete vortices in the outer region (Figs. 13, 14), (2) formation of vortex-like motions in the inner region wall boundary layer (Fig. 14b), (3) separation of the wall jet from the top surface due to an adverse pressure gradient and (4) interaction of the separated wall jet with the global recirculation cell. Neither the vortex pairing in the inner and outer region nor the re-laminarization could be distinguished from the PIV measurements. The different observations are likely caused by the differences in experimental setup: the measurements in this study are performed in a confined space with an influence of the recirculation zone on the flow physics of the wall jet, whereas the measurements by Bajura and Catalano (1975) were conducted in an unconfined space, without influence of an opposing wall on the transition process. Furthermore, the measurement resolution and frequency in our study might have been too low to capture the pairing of vortices in the test section. 
In addition, there are some other limitations concerning the experimental work described in this paper. First, the reflections of the glass bottom of the test section made it impossible to analyze the flow in this area of the cube. As a result, some information on the flow pattern is lacking, although one must note that the bottom of the cube is not the primary area of interest in this study, in contrast to the wall jet region. Second, the determination of the Strouhal number has not been conducted using FFT, although the suitability of the used method has been assessed using FFT. Future work should consist of measurements with a higher temporal resolution (time-resolved measurements) to enable the use of more accurate methods to determine the Strouhal number. Finally, there appears to be a small discrepancy between the velocity profiles obtained from the higher resolution measurements in the vicinity of the inlet and the profiles obtained from the measurements in the entire cross-section of the cube. The second set of profiles (Fig. 6) indicates the presence of a top-hat profile, which is not that clearly visible in the profiles obtained from the second set of measurements (Fig. 10). Furthermore, the values of $U / U_{M}$ below the entrance of the wall jet show small differences between ROI1 and ROI2. A possible explanation for these small deviations is the lower measuring resolution for the measurements in the entire crosssection of the cube (ROI1), in combination with relatively smaller seeding due to the larger field of view.

This study is a first step in a more extensive research project on transitional wall jets in a confined space. Future work will include measurements for different inlet opening heights $h$, inlet geometries and additional values of the slot Reynolds number $R e=U_{0} h / v$. Alongside the PIV measurements, point measurements will be conducted using Laser Doppler Anemometry (LDA). These point measurements in an air filled setup $\left(2 \times 2 \times 2 \mathrm{~m}^{3}\right)$ will provide time-resolved data of the air flow pattern, which will provide valuable complementary information to the data set presented in this paper. In addition, LDA is more suited than PIV to carry out a local study concerning wall jet detachment, especially its intermittency (Moureh and Flick 2003) and the local turbulence anisotropy (Moureh and Flick 2005).

\section{Conclusions}

This paper presents a detailed and systematic experimental analysis of a transitional plane wall jet in a confined space. To ensure that the measurements are conducted for a transitional flow regime, flow visualizations have been performed using fluorescent dye. Based on the flow visualizations, PIV measurements have been conducted for seven Reynolds numbers, ranging from 800 to 2,500. For each value of $R e$, two sets of measurements have been obtained: one of the flow pattern in the entire cross-section of the cube (region of interest $=L \times L$ ) and one with a smaller region of interest near the inlet $(0.6 L \times 0.4 L)$ to increase the measurement resolution in this area with large velocity gradients. Both the time-averaged and the instantaneous vector fields have been analyzed.

From the time-averaged results, the following conclusions can be made:

- The flow visualizations and the PIV measurements have indicated that the onset of jet instability occurs further downstream as $R e$ increases.

- The general flow pattern is the same for all tested $R e$-values $(800<R e<2,500)$. The wall jet drives the large recirculation cell in the center of the cube. Smaller recirculation cells are present in the downstream top corner and just below the jet entrance in the enclosure.

- The size of these small recirculation cells decreases with increasing $R e$.

- The velocity profiles show a clear $R e$-dependency that increases with increasing distance from the inlet.

- Jet detachment and the location of maximum jet velocity $\left(y_{C}\right)$ both depend on $R e$; the jet detachment occurs further downstream and $y_{C}$ increases with increasing $R e$.

- The wall jet is not yet fully developed at small distances from the inlet, while at larger distances from the inlet, the confinement of the jet and the associated adverse pressure gradient lead to flow separation and a reversed flow.

- The outer region of the measured wall jet resembles the theoretical values for a laminar wall jet for all tested $R e$-values. The inner region for $R e \approx 1,000$ also shows a fair to good agreement with the laminar wall jet. For $R e \approx 1,750$ and $R e \approx 2,500$, however, the velocity profiles in the inner region are shifting toward the theoretical values of a turbulent wall jet.

- Below the wall jet region, the dimensionless vorticity profiles are $R e$-independent, whereas in the wall jet region, the values of $\omega_{z}$ do not overlap, but also do not show a trend with $R e$.

- The large velocity gradients in the inner and outer region of the wall jet due to the boundary layer and the shear layer result in increased values of positive and negative $z$-vorticity, respectively. The weak negative vorticity in the center of the test section is associated with the large clockwise-rotating recirculation cell, and the uniform $\omega_{z}$-value indicates a solid-body rotation in this cell.

Analysis of the instantaneous velocity vector fields has led to the following conclusions: 
- Kelvin-Helmholtz-type instability waves are present at the bottom part of the wall jet, which grow and lead to the formation of discrete vortical structures in the outer region.

- The $Q$ criterion shows that the positive vorticity in the inner region, and before jet detachment, is the result of shear in the boundary layer (hyperbolic region). However, in the outer region, a vortex train is present with alternating regions of $Q<0$ and $Q>0$, indicating the presence of rotation-dominated regions (elliptic flow) and strain-dominated regions (hyperbolic flow), respectively.

- The instantaneous velocity profiles indicate a lower velocity in the outer region of the wall jet at the locations of the vortical structures. This feature is due to the presence of clockwise-rotating coherent structures, the bottom of which causes the locally lower values of the instantaneous velocity.

- The number of formed vortices per unit length increases with $R e$, and this increase is larger than the increase in inlet velocity; therefore, the Strouhal number $S t$ increases with an increase in $R e$.

Acknowledgments Twan van Hooff is currently a PhD student funded by both Eindhoven University of Technology in the Netherlands and Fonds Wetenschappelijk Onderzoek (FWO)-Flanders, Belgium (FWO project number: G.0435.08). The FWO Flanders supports and stimulates fundamental research in Flanders. Its contribution is gratefully acknowledged. Thijs Defraeye is a postdoctoral fellow of the FWO-Flanders and acknowledges its support. The measurements reported in this paper were supported by the Laboratory of the Unit Building Physics and Services (BPS) at Eindhoven University of Technology and the Laboratory of Building Physics at the Katholieke Universiteit Leuven. Special thanks go to Jan Diepens, head of LBPS, and Wout van Bommel, Harrie Smulders, Geert-Jan Maas and Peter Cappon, members of the LBPS, for their valuable contributions.

Open Access This article is distributed under the terms of the Creative Commons Attribution License which permits any use, distribution, and reproduction in any medium, provided the original author(s) and the source are credited.

\section{References}

Ahlman D, Velter G, Brethouwer G, Johansson AV (2009) Direct numerical simulation of nonisothermal turbulent wall jets. Phys Fluids 21:035101

Amitay M, Cohen J (1997) Instability of a two-dimensional plane wall jet subjected to blowing or suction. J Fluid Mech 344:67-94

Angioletti M, Di Tomasso RM, Nino E, Ruocco G (2003) Simultaneous visualization of flow field and evaluation of local heat transfer by transitional impinging jets. Int J Heat Mass Transfer 46:1703-1713

Awbi HB (2003) Ventilation of buildings. Spon Press, London

Bajura RA, Catalano MR (1975) Transition in a two-dimensional plane wall jet. J Fluid Mech 70:773-799

Bakke P (1957) An experimental investigation of a wall jet. J Fluid Mech 2:467-472
Balabel A, El-Askary WA (2011) On the performance of linear and nonlinear $\mathrm{k}-\varepsilon$ turbulence models in various jet flow applications. Eur J Mech B Fluids 30:325-340

Beavers GS, Wilson TA (1970) Vortex growth in jets. J Fluid Mech 44:97-112

Bhattacharjee P, Loth E (2004) Simulations of laminar and transitional cold wall jets. Int J Heat Fluid Flow 25:32-43

Bradshaw P, Gee MT (1960) Turbulent wall jets with and without an external stream. Aeronautical Research Council R\&M 3252

Chen Q (2009) Ventilation performance prediction for buildings: A method overview and recent applications. Build Environ 44:848-858

Cieślik AR, Kamp LPJ, Clercx HJH, van Heijst GJF (2010) Threedimensional structures in a shallow flow. J Hydro-Environ Res 4:89-101

Clayton BR, Massey BS (1967) Flow visualization in water: a review of techniques. J Sci Instrum 44:2-11

Coleman HW, Steel WG (1999) Experimentation and uncertainty analysis for engineers. Wiley, New York

Conlon BP, Lichter S (1995) Dipole formation in the transient planar wall jet. Phys Fluids 7:999-1014

Davidson L, Nielsen PV, Topp C (2000) Low Reynolds number effects in ventilated rooms: a numerical study. Proc. of Roomvent 2000, Oxford: 307-312

Eriksson JG, Karlsson RI, Persson J (1998) An experimental study of a two-dimensional plane turbulent wall jet. Exp Fluids 25:50-60

Etheridge DW, Sandberg M (1996) Building ventilation: theory and measurement. Wiley, London

Förthmann E (1934) Über turbulente strahleausbreitung. Ing Archiv $5: 42-54$

Glauert P (1956) The wall jet. J Fluid Mech 1:625-643

Gogineni S, Shih C (1997) Experimental investigation of the unsteady structure of a transitional plane wall jet. Exp Fluids 23:121-129

Gogineni S, Visbal M, Shih C (1999) Phase-resolved PIV measurements in a transitional plane wall jet: a numerical comparison. Exp Fluids 27:126-136

Hsiao FB, Sheu SS (1994) Double row vortical structures in the near field region of a plane wall jet. Exp Fluids 17:291-301

Hsiao FB, Sheu SS (1996) Experimental studies on flow transition of a plane wall jet. Aeronaut J100(999):373-380

Isern-Fontanet J, Font J, García-Ladona E, Emelianov M, Millot C, Taupier-Letage I (2004) Spatial structures of anticyclonic eddies in the Algerian basin (Mediterranean Sea) analyzed using the Okubo-Weiss parameter. Deep Sea Res II 51:3009-3028

Keane RD, Adrian RJ (1990) Optimization of particle image velocimeters. Part I: double pulsed systems. Meas Sci and Technol 1:1202-1215

Konijnenberg JA, Andersson HI, Billdal JT, van Heijst GJF (1994) Spin-up in a rectangular tank with low angular velocity. Phys Fluids 6:1168-1176

Kwon SJ, Seo IW (2005) Reynolds number effects on the behavior of a non-buoyant round jet. Exp Fluids 38:801-812

Launder BE, Rodi W (1981) The turbulent wall jet. Prog Aerospace Sci 19:81-128

Launder BE, Rodi W (1983) The turbulent wall jet—measurements and modelling. Annu Rev Fluid Mech 15:429-459

Lemieux GP, Oosthuizen PH (1985) Experimental study of the behavior of plane turbulent jets at low Reynolds numbers. AIAA J 23:1845-1846

Lichter S, Flór JB, van Heijst GJF (1992) Modelling the separation and eddy formation of coastal currents in a stratified tank. Exp Fluids 13:11-16

Molenaar D, Clercx HJH, van Heijst GJF (2004) Angular momentum of forced 2D turbulence in a square no-slip domain. Phys D 196:329-340 
Moureh J, Flick D (2003) Wall air-jet characteristics and airflow patterns within a slot ventilated enclosure. Int $\mathrm{J}$ Therm Sci 42:703-711

Moureh J, Flick D (2005) Airflow characteristics within a slotventilated enclosure. Int J Heat Fluid Flow 26:12-24

Mumford JC (1982) The structure of the large scale eddies in fully developed turbulent shear flows. Part 1. The plane jet. J Fluid Mech 116:241-268

Namer I, Ötügen MV (1988) Velocity measurements in a plane turbulent air jet at moderate Reynolds numbers. Exp Fluids 6:387-399

Nielsen PV (1974) Flow in air conditioned rooms, PhD Thesis, Technical University of Denmark, Copenhagen

Nielsen PV, Filholm C, Topp C, Davidson L (2000) Model experiments with low Reynolds number effects in a ventilated room. Proc. of Roomvent, Oxford: 185-190

O'Neill P, Soria J, Honnery D (2004) The stability of low Reynolds number round jets. Exp Fluids 36:473-483

Okubo A (1970) Horizontal dispersion of floatable trajectories in the vicinity of velocity singularities such as convergencies. DeepSea Res 17:445-454

Prasad AK (2000) Particle image velocimetry. Curr Sci 79:51-60

Sato H (1960) The stability and transition of a two-dimensional jet. J Fluid Mech 7:53-80

Sato H, Sakao F (1964) An experimental investigation of the instability of a two-dimensional jet at low Reynolds numbers. J Fluid Mech 20:337-352

Schwarz WH, Cosart WP (1961) The two-dimensional wall jet. J Fluid Mech 10:481-495

Sigalla A (1958) Measurements of skin friction in a plane turbulent wall jet. J Royal Aero Soc 62:873-877

Suresh PR, Srinivasan K, Sundararajan T, Das SK (2008) Reynolds number dependence of plane jet development in the transitional regime. Phys Fluids 20:044105-044105-12
Todde V, Spazzini PG, Sandberg M (2009) Experimental analysis of low-Reynolds number free jets: evolution along the jet centerline and Reynolds number effects. Exp Fluids 47:279-294

Topp C, Nielsen PV, Davidson L (2000) Room airflows with low Reynolds number effects. Proc. of Roomvent, Oxford: 541-546

van Heijst GJF, Davies PA, Davis RG (1990) Spin-up in a rectangular container. Phys Fluids 2:150-159

van Hooff T, Blocken B, Defraeye T, Carmeliet J, van Heijst GJF (2012) PIV measurements and analysis of transitional flow in a reduced-scale model: ventilation by a free plane jet with Coanda effect. Build Environ. http://dx.doi.org/10.1016/j.buildenv.2012. 03.020

Verhoff A (1963) The two-dimensional turbulent wall jet with and without an external stream Report 626. Princeton University, New Jersey

Vosbeek PWC, van Geffen JHGM, Meleshko VV, van Heijst GJF (1997) Collapse interactions of finite-sized two-dimensional vortices. Phys Fluids 9:3315

Wang M, Chen Q (2009) Assessment of various turbulence models for transitional flows in enclosed environment. HVAC\&R Res 15:1099-1119

Wang M, Chen Q (2010) On a hybrid RANS/LES approach for indoor airflow modelling. HVAC\&R Res 16:731-747

Weiss J (1991) The dynamics of enstrophy transfer in twodimensional hydrodynamics. Phys D 48:273-294

Wells MG, Clercx HJH, van Heijst GJF (2007) Vortices in oscillating spin-up. J Fluid Mech 573:339-369

Wernz S, Fasel HF (2007) Nonlinear resonances in a laminar wall jet: ejection of dipolar vortices. J Fluid Mech 588:279-308

Wygnanski I, Katz Y, Horev E (1992) On the applicability of various scaling laws to the turbulent wall jet. J Fluid Mech 234:669-690 